\title{
EXPONENTIAL POLYNOMIAL INEQUALITIES AND MONOMIAL SUM INEQUALITIES IN p-NEWTON SEQUENCES
}

\author{
Charles R. Johnson, Williamsburg, \\ Carlos Marijuán, Miriam Pisonero, Valladolid, \\ Michael Yeh, West Lafayette
}

(Received December 18, 2015)

\section{Dedicated to the memory of Miroslav Fiedler}

Abstract. We consider inequalities between sums of monomials that hold for all p-Newton sequences. This continues recent work in which inequalities between sums of two, two-term monomials were combinatorially characterized (via the indices involved). Our focus is on the case of sums of three, two-term monomials, but this is very much more complicated. We develop and use a theory of exponential polynomial inequalities to give a sufficient condition for general monomial sum inequalities, and use the sufficient condition in two ways. The sufficient condition is necessary in the case of sums of two monomials but is not known if it is for sums of more. A complete description of the desired inequalities is given for Newton sequences of less than 5 terms.

Keywords: exponential polynomial; Newton inequality; Newton coefficients; p-Newton sequence

MSC 2010: 15A15, 15A45, 11C20, 15A 18

\section{INTRODUCTION}

An ordered (not necessarily numerically) sequence

$$
c: c_{0}, c_{1}, c_{2}, \ldots, c_{n}
$$

of real numbers is called a Newton sequence if

$$
c_{i-1} c_{i+1} \leqslant c_{i}^{2}
$$

Partially supported by MTM2015-365764-C-1-P (MINECO-FEDER), MTM2010-19281-

C03-01 and NSF grant \#DMS-0751964. 
for $1 \leqslant i \leqslant n-1$. Each of these inequalities is called a Newton inequality, cf. [4]. See [2], [3], [1] for general background on Newton sequences, how they arise, the relationship to matrices and prior results. A Newton sequence is called $p$-Newton if each of the $c_{i}$ 's is positive. For these sequences, Newton inequalities can be generalized as

$$
c_{i-k} c_{j+k} \leqslant c_{i} c_{j}
$$

for $0 \leqslant i \leqslant j \leqslant n$ and $0 \leqslant k \leqslant \min \{i, n-j\}$, see [2], Lemma 9. In fact, for $p \leqslant q$ and $r \leqslant s$ nonnegative integers with $p+q=r+s$ we have

$$
c_{r} c_{s} \leqslant c_{p} c_{q} \Leftrightarrow r \leqslant p \leqslant q \leqslant s .
$$

A positive sequence is p-Newton if and only if its sequence of ratios of consecutive terms $c_{i+1} / c_{i}$ is nonincreasing.

For any p-Newton sequence

$$
c_{0}, c_{1}, \ldots, c_{n}
$$

and any $r>1$, we may re-write the $c$ 's as

$$
c_{i}=r^{x_{i}}
$$

$i=0, \ldots, n$, to obtain the Newton exponent sequence (NES)

$$
x_{0}, x_{1}, \ldots, x_{n}
$$

Clearly, we have a NES if and only if

$$
x_{i-1}+x_{i+1} \leqslant 2 x_{i},
$$

$i=1, \ldots, n-1$.

By a monomial in a p-Newton sequence, we mean an expression of the form

$$
c^{a}:=c_{0}^{a_{0}} c_{1}^{a_{1}} \ldots c_{n}^{a_{n}} .
$$

The length of $c^{a}$ is the number $a_{0}+a_{1}+\ldots+a_{n}$, and the weight of $c^{a}$ is the sum of the indices weighted by their exponents in the monomial. In [3], all inequalities between pairs of monomials that hold for all p-Newton sequences were characterized. Here we continue recent work [1] on inequalities between sums of simple monomials (all exponents are nonnegative integers) in p-Newton sequences. Even in the most elemental cases, there are inequalities in which term-wise domination of monomials does not occur. 
We may write the $h$-th summand (of a sum of simple monomials) as

$$
c^{a(h)}=c_{i_{h 1}} c_{i_{h 2}} \ldots c_{i_{h k_{h}}}
$$

in which repeated subscripts are allowed and, of course, some may not appear. Our sum of monomials may then be written as

$$
c^{a, m}=\sum_{h=1}^{m} c^{a(h)},
$$

which is fully described by $m$ index lists, the subscripts appearing in each monomial.

We are interested in inequalities between two such monomial sums, i.e., when we have

$$
c^{a, m} \leqslant c^{b, m}
$$

for all p-Newton sequences $c$. In [1], we characterized the case $m=2$ and $k_{h}=2$, $h=1,2$ in a combinatorial way. This is the case 22 versus 22 , i.e., 2 monomials with length 2 on each side. The case 222 versus $222(m=3$, length 2 in each monomial) is discussed here, but it proves dramatically more complicated. We are able to give a complete account of all inequalities for p-Newton sequences of fewer than 5 terms, and we have summarized them in a characterization theorem in Section 4.

We also develop a theory of inequalities between $n$-term exponential polynomials (Section 2). By considering the exponents of a p-Newton sequence, relative to a positive base $r$, we are able to use this in Section 3 to give a general sufficient condition for monomial sum inequalities in p-Newton sequences. This is used to verify some monomial sum inequalities, and to show, by example, in Section 5 , that for longer p-Newton sequences (more than 4 terms) there are monomial sum inequalities in the 222 versus 222 case that are rather different in character from the 4 -term ones. Finally, we include an Addendum completing the proof of Lemma 19 given in [1].

For reference, we re-write (1.6) in extended form

$$
\begin{aligned}
c_{j_{11}} c_{j_{12}} \ldots c_{j_{1 l_{1}}}+\ldots+ & c_{j_{m 1}} c_{j_{m 2}} \ldots c_{j_{m l_{m}}} \\
& \geqslant c_{i_{11}} c_{i_{12}} \ldots c_{i_{k_{1}}}+\ldots+c_{i_{m 1}} c_{i_{m 2}} \ldots c_{i_{m k_{m}}} .
\end{aligned}
$$

We may assume, for convenience, without loss of generality, that

$$
\begin{aligned}
& i_{11} \leqslant i_{12} \leqslant \ldots \leqslant i_{1 k_{1}}, \ldots, i_{m 1} \leqslant i_{m 2} \leqslant \ldots \leqslant i_{m k_{m}}, \\
& j_{11} \leqslant j_{12} \leqslant \ldots \leqslant j_{1 l_{1}}, \ldots, j_{m 1} \leqslant j_{m 2} \leqslant \ldots \leqslant j_{m l_{m}} .
\end{aligned}
$$


Since the all 1's sequence is p-Newton, the right hand side must have as many monomials as the left hand side, and it is natural to assume the extreme case of the same number. Several observations have been made [3], [1] about where the larger and smaller indices can occur: the highest index is on the smaller side, the lowest index is on the larger side, ...

\section{EXPONENTIAL POLYNOMIAL INEQUALITIES}

An exponential polynomial is a function from $\mathbb{R}_{+}$to $\mathbb{R}$ of the form

$$
r^{p_{1}}+r^{p_{2}}+\ldots+r^{p_{n}}
$$

in which $r>0$ is a variable and $p_{1}, \ldots, p_{n}$ are given real exponents. If

$$
r^{p_{1}}+r^{p_{2}}+\ldots+r^{p_{n}} \geqslant r^{q_{1}}+r^{q_{2}}+\ldots+r^{q_{n}}
$$

for all $r \geqslant 1$, we say that the real $n$-list $p \quad r$-dominates the real $n$-list $q$ and write

$$
p \geqslant r q \text {. }
$$

The notion " $\geqslant_{r}$ " does not depend upon how the $p$ 's and $q$ 's are ordered, but we usually write them in descending order. The list $p$ is said to weakly majorize the list $q$ if

$$
\sum_{i=1}^{k} p[i] \geqslant \sum_{i=1}^{k} q[i]
$$

for $k=1, \ldots, n$, in which $p[i](q[i])$ denotes the $i$-th largest of the $p$ 's $(q$ 's $)$, in case they were not already in descending order. We write $p \succ_{w} q$. In case there is equality for $k=n$, the list $p$ is said to majorize the list $q$, written $p \succ q$.

We raise the question of exactly when $p \geqslant_{r} q$. In general, this seems subtle, but it is useful to us in understanding inequalities between monomial sums on p-Newton sequences. In [1], it was noted that:

Theorem 2.1. For $n=2, p \geqslant_{r} q$ if and only if $p$ weakly majorizes $q$.

However, it was also noted that $\geqslant_{r}$ in a greater variety of ways for larger $n$.

Example 2.2. The polynomial

$$
f(r)=r^{10}+r^{6}+r^{5}-r^{9}-r^{8}-r^{4}
$$

satisfies $f(r)>0$ for $r>1$, as

$$
f(r)=(r-1)^{3} r^{4}\left(r^{3}+2 r^{2}+2 r+1\right) .
$$

But, as $10+6<9+8,(10,6,5)$ does not weakly majorize $(9,8,4)$. 
Here, we record what we know about $r$-domination (for exponential polynomials).

Lemma 2.3. Let $p$ and $q$ be real $n$-lists. If

$$
\sum_{i=1}^{n} p_{i}^{k} \geqslant \sum_{i=1}^{n} q_{i}^{k}
$$

for all integers $k \geqslant 0$, then $p \geqslant_{r} q$.

P r o of. Using the Maclaurin series for $\mathrm{e}^{z}$, we find that

$$
\sum_{i=1}^{n} r^{x_{i}}=\sum_{i=1}^{n} \mathrm{e}^{x_{i} \log r}=\sum_{k=0}^{\infty} \frac{\log ^{k} r}{k !}\left(x_{1}^{k}+\ldots+x_{n}^{k}\right)
$$

for $r \geqslant 1$. Because $\log r \geqslant 0$ for $r \geqslant 1$, we get

$$
\sum_{k=0}^{\infty} \frac{\log ^{k} r}{k !}\left(p_{1}^{k}+\ldots+p_{n}^{k}\right) \geqslant \sum_{k=0}^{\infty} \frac{\log ^{k} r}{k !}\left(q_{1}^{k}+\ldots+q_{n}^{k}\right)
$$

for all $r \geqslant 1$, from which the result follows.

It is not clear how many $k$ 's are needed to verify the hypothesis of Lemma 2.3.

Example 2.4. Observe that $p_{1}^{k}+\ldots+p_{n}^{k} \geqslant q_{1}^{k}+\ldots+q_{n}^{k}$ for $0 \leqslant k \leqslant n$ does not imply that $p_{1}^{k}+\ldots+p_{n}^{k} \geqslant q_{1}^{k}+\ldots+q_{n}^{k}$ for all integers $k \geqslant 0$. A counterexample is $p=(10,7.7,3.5)$ and $q=(9.99,7.755,1)$. In fact, $\sum p_{i}^{k} \geqslant \sum q_{i}^{k}$ for $0 \leqslant k \leqslant 5$, but $\sum p_{i}^{6}<\sum q_{i}^{6}$.

We can now see that majorization is sufficient for $r$-domination. As seen in Example 2.2 , it is not necessary for $n \geqslant 3$.

Theorem 2.5. For real $n$-sequences $p$ and $q$, if $p \succ_{w} q$, then $p \geqslant_{r} q$.

P r o of. Without loss of generality, we may assume that $p$ and $q$ are $n$-lists written in descending order. Fix $r \geqslant 1$ and define $g: \mathbb{R}^{n} \rightarrow \mathbb{R}$ by $g(x)=r^{x_{1}}+\ldots+r^{x_{n}}$. Because $g$ is differentiable, by the Mean value theorem there exists a $\lambda \in(0,1)$ such that

$$
\begin{aligned}
g(p)-g(q) & =\operatorname{Dg}((1-\lambda) p+\lambda q)(p-q) \\
& =\log r \sum_{i=1}^{n} r_{i}\left(p_{i}-q_{i}\right),
\end{aligned}
$$


where $r_{i}=r^{(1-\lambda) p_{i}+\lambda q_{i}}$. Using the fact that $\log r \geqslant 0, r_{i} \geqslant r_{i+1} \geqslant 0$ for all $i, p \succ_{w} q$, and $p$ and $q$ are in descending order,

$$
\begin{aligned}
(*) & =\log r\left(\sum_{i=1}^{n-1} r_{i}\left(p_{i}-q_{i}\right)+r_{n}\left(p_{n}-q_{n}\right)\right) \\
& \geqslant \log r\left(\sum_{i=1}^{n-1} r_{i}\left(p_{i}-q_{i}\right)+\sum_{i=1}^{n-1} r_{n}\left(q_{i}-p_{i}\right)\right) \\
& =\log r \sum_{i=1}^{n-1}\left(r_{i}-r_{n}\right)\left(p_{i}-q_{i}\right) \\
& \geqslant \log r\left(\sum_{i=1}^{n-2}\left(r_{i}-r_{n}\right)\left(p_{i}-q_{i}\right)+\sum_{i=1}^{n-2}\left(r_{n-1}-r_{n}\right)\left(q_{i}-p_{i}\right)\right) \\
& =\log r \sum_{i=1}^{n-2}\left(r_{i}-r_{n-1}\right)\left(p_{i}-q_{i}\right) \\
& \geqslant \ldots \geqslant \log r \sum_{i=1}^{1}\left(r_{i}-r_{2}\right)\left(p_{i}-q_{i}\right) \\
& =(\log r)\left(r_{1}-r_{2}\right)\left(p_{1}-q_{1}\right) \geqslant 0 .
\end{aligned}
$$

But $r \geqslant 1$ was arbitrary, and the result follows.

In spite of Example 2.2, we can characterize $\geqslant_{r}$ when $n=3$, and the characterization is just majorization when $n=3$ and $p_{1}+p_{2}+p_{3}=q_{1}+q_{2}+q_{3}$, if we ask that the inequality hold for $r>0$.

Lemma 2.6. Let $p, q \in \mathbb{R}^{3}$ and define $f: \mathbb{R}_{+} \rightarrow \mathbb{R}$ by

$$
f(r)=r^{p_{1}}+r^{p_{2}}+r^{p_{3}}-r^{q_{1}}-r^{q_{2}}-r^{q_{3}} .
$$

Then the following statements are equivalent:

(B) $p_{1}^{n}+p_{2}^{n}+p_{3}^{n}=q_{1}^{n}+q_{2}^{n}+q_{3}^{n}$ for all integers $n \geqslant 1$ or $p_{1}^{N}+p_{2}^{N}+p_{3}^{N}>q_{1}^{N}+q_{2}^{N}+q_{3}^{N}$, where $N=\min \left\{n \in \mathbb{N}: p_{1}^{n}+p_{2}^{n}+p_{3}^{n} \neq q_{1}^{n}+q_{2}^{n}+q_{3}^{n}\right\}$.

$\left(\mathrm{B}^{\prime}\right) f^{(n)}(1)=0$ for all integers $n \geqslant 1$ or $f^{(N)}(1)>0$, where $N=\min \{n \in \mathbb{N}$ : $\left.f^{(n)}(1) \neq 0\right\}$.

Pr o of. Let $n \in \mathbb{N}$. An easy computation shows that

$$
\prod_{i=0}^{n-1}(x-i)=x^{n}+a_{n-1}^{(n)} x^{n-1}+\ldots+a_{1}^{(n)} x
$$


for some constants $a_{j}^{(n)}$. Therefore, $f^{(n)}(1)$ has the form

$$
f^{(n)}(1)=\sum_{j=1}^{n} a_{j}^{(n)}\left(p_{1}^{j}+p_{2}^{j}+p_{3}^{j}-q_{1}^{j}-q_{2}^{j}-q_{3}^{j}\right), \quad a_{n}^{(n)}=1
$$

If $p_{1}^{n}+p_{2}^{n}+p_{3}^{n}=q_{1}^{n}+q_{2}^{n}+q_{3}^{n}$ for all $n$, then it is clear from $(*)$ that $f^{(n)}(1)=0$ for all $n$. Now, assume that $f^{(n)}(1)=0$ for all $n$. When $n=1$, this means that $p_{1}+p_{2}+p_{3}=q_{1}+q_{2}+q_{3}$. Suppose we know that $p_{1}^{n}+p_{2}^{n}+p_{3}^{n}=q_{1}^{n}+q_{2}^{n}+q_{3}^{n}$ for $1 \leqslant n \leqslant k$. Then from $(*), 0=f^{(k+1)}(1)=p_{1}^{k+1}+p_{2}^{k+1}+p_{3}^{k+1}-q_{1}^{k+1}-q_{2}^{k+1}-q_{3}^{k+1}$. By induction, $p_{1}^{n}+p_{2}^{n}+p_{3}^{n}=q_{1}^{n}+q_{2}^{n}+q_{3}^{n}$ for all $n$.

If $p_{1}^{n}+p_{2}^{n}+p_{3}^{n} \neq q_{1}^{n}+q_{2}^{n}+q_{3}^{n}$ for some $n$, let $N=\min \left\{n \in \mathbb{N}: p_{1}^{n}+p_{2}^{n}+p_{3}^{n} \neq\right.$ $\left.q_{1}^{n}+q_{2}^{n}+q_{3}^{n}\right\}$ and suppose that $p_{1}^{N}+p_{2}^{N}+p_{3}^{N}>q_{1}^{N}+q_{2}^{N}+q_{3}^{N}$. From (*) and the definition of $N$, we find that $0<p_{1}^{N}+p_{2}^{N}+p_{3}^{N}-q_{1}^{N}-q_{2}^{N}-q_{3}^{N}=f^{(N)}(1)$ and that $f^{(n)}(1)=0$ for $n<N$, i.e. $N=\min \left\{n \in \mathbb{N}: f^{(n)}(1) \neq 0\right\}$ and $f^{(N)}(1)>0$. On the other hand, if $f^{(n)}(1) \neq 0$ for some $n$, let $N=\min \left\{n \in \mathbb{N}: f^{(n)}(1) \neq 0\right\}$ and suppose that $f^{(N)}(1)>0$. If $N=1$, then $p_{1}+p_{2}+p_{3}>q_{1}+q_{2}+q_{3}$ and we are done. Otherwise, a finite induction argument shows that $f^{(n)}(1)=p_{1}^{n}+p_{2}^{n}+p_{3}^{n}-q_{1}^{n}-q_{2}^{n}-q_{3}^{n}$ for $n \leqslant N$. Therefore, $p_{1}^{N}+p_{2}^{N}+p_{3}^{N}>q_{1}^{N}+q_{2}^{N}+q_{3}^{N}$ and $N=\min \left\{n \in \mathbb{N}: p_{1}^{n}+p_{2}^{n}+p_{3}^{n} \neq\right.$ $\left.q_{1}^{n}+q_{2}^{n}+q_{3}^{n}\right\}$.

Now we can give our characterization for general real 3-sequences.

Theorem 2.7. Suppose that $p_{1} \geqslant p_{2} \geqslant p_{3}$ and $q_{1} \geqslant q_{2} \geqslant q_{3}$ are real 3-sequences satisfying $p_{1}+p_{2}+p_{3}=q_{1}+q_{2}+q_{3}$. Then $p \geqslant_{r} q$ if and only if

(A) $p_{1} \geqslant q_{1}$ and

(B) $p_{1}^{n}+p_{2}^{n}+p_{3}^{n}=q_{1}^{n}+q_{2}^{n}+q_{3}^{n}$ for all integers $n \geqslant 1$ or $p_{1}^{N}+p_{2}^{N}+p_{3}^{N}>q_{1}^{N}+q_{2}^{N}+q_{3}^{N}$, where $N=\min \left\{n \in \mathbb{N}: p_{1}^{n}+p_{2}^{n}+p_{3}^{n} \neq q_{1}^{n}+q_{2}^{n}+q_{3}^{n}\right\}$.

Pr o of. To prove the theorem, we will show that $f(r) \geqslant 0$ for all $r \geqslant 1$ if and only if $(\mathrm{A})$ and $\left(\mathrm{B}^{\prime}\right)$ hold, where $f$ and $\left(\mathrm{B}^{\prime}\right)$ are as in Lemma 2.6.

$(\Rightarrow)$ If $p_{1}<q_{1}$, then $f(r)<0$ eventually. On the other hand, if $f^{(n)}(1) \neq 0$ for some $n$ and $f^{(N)}(1)<0$, where $N=\min \left\{n \in \mathbb{N}: f^{(n)}(1) \neq 0\right\}$, then $f<0$ on $(1,1+\varepsilon)$ for some $\varepsilon>0$.

$(\Leftarrow)$ If $f^{(n)}(1)=0$ for all $n$, then since $f$ is analytic, $f \equiv 0$ in a neighborhood of 1 . Recall that a nonzero analytic function on an open connected set has isolated zeroes. Hence, $f \equiv 0$.

So, suppose that $f^{(n)}(1) \neq 0$ for some $n$, and let $N=\min \left\{n \in \mathbb{N}: f^{(n)}(1) \neq 0\right\}$. Then $f^{(N)}(1)>0$, and $f>0$ on $(1,1+\varepsilon)$ for some $\varepsilon>0$. In particular, we have $q \nsucc p$. 
If $p_{3} \leqslant q_{3}$, then $p_{1}+p_{2} \geqslant q_{1}+q_{2}$ and $p \succ q$, which is sufficient by Theorem 2.5. If $p_{3}>q_{3}$, then $p_{1}+p_{2}<q_{1}+q_{2}, p_{2}<q_{2}$, and therefore

$$
p_{1}>q_{1} \geqslant q_{2}>p_{2} \geqslant p_{3}>q_{3} .
$$

Note that $p_{1} \neq q_{1}$ since $q \nsucc p$. The function $f$ has 3 sign changes, so Descartes' rule of signs, see [5], implies that $f$ has 1 or 3 positive roots (counted with multiplicity). There are 3 roots, $z_{1}=z_{2}=1$ and $z_{3}$. If $z_{3}>1$, then (i) $f>0$ on $(1,1+\varepsilon)$ implies that $f>0$ on $\left(1, z_{3}\right)$, (ii) since $z_{3}$ is a simple root, $f<0$ on $\left(z_{3}, z_{3}+\eta\right)$ for some $\eta>0$, and (iii) because $f$ is continuous and $f>0$ eventually, $f$ has another root $z_{4}>z_{3}$. This is a contradiction. Since $f>0$ on $(1,1+\varepsilon)$ and has no roots greater than $1, f(r) \geqslant 0$ for all $r \geqslant 1$.

Note that in Example 2.2 we have $p \geqslant_{r} q$, condition (A) from the previous theorem is clear, and condition (B) from the previous theorem is satisfied for $N=3$.

Since the derivative of $r_{n}^{p_{1}}+\ldots+r^{p_{n}}$ with respect to $r$ at $r=1$ is $\sum_{i=1}^{n} p_{i}, p \geqslant_{r} q$ implies that $\sum_{i=1}^{n} p_{i} \geqslant \sum_{i=1}^{n} q_{i}$. Also, $p \geqslant r q$ implies that $p_{1} \geqslant q_{1}$, because of large $r$ (and $p_{2} \geqslant q_{2}$ if $p_{1}=q_{1}$, etc). If we now ask that

$$
r^{p_{1}}+r^{p_{2}}+r^{p_{3}} \geqslant r^{q_{1}}+r^{q_{2}}+r^{q_{3}}
$$

for all $r \geqslant 0$, there is a nice answer. Effectively, the interval $r \in(0,1)$ negates the $p$ 's and $q$ 's.

Theorem 2.8. If $p$ and $q$ are real 3-sequences, then

$$
r^{p_{1}}+r^{p_{2}}+r^{p_{3}} \geqslant r^{q_{1}}+r^{q_{2}}+r^{q_{3}}
$$

for all $r>0$ if and only if $p \succ q$.

P r o o f. Without loss of generality, we may assume that $p$ and $q$ are $n$-lists written in descending order.

$(\Rightarrow)$ Let $f$ be the function of Lemma 2.6. Then certainly $f(r) \geqslant 0$ for all $r \geqslant 1$, so it is necessary that $p_{1} \geqslant q_{1}$ and $p_{1}+p_{2}+p_{3}-q_{1}-q_{2}-q_{3}=f^{\prime}(1) \geqslant 0$ (since $f(1)=0)$. Let $\tilde{f}: \mathbb{R}^{+} \rightarrow \mathbb{R}, \tilde{f}(s)=s^{-p_{1}}+s^{-p_{2}}+s^{-p_{3}}-s^{-q_{1}}-s^{-q_{2}}-s^{-q_{3}}$ so that $\tilde{f}(s)=f(1 / s)$. Hence, $\tilde{f}(s) \geqslant 0$ for all $s \geqslant 1$, and as above, it is necessary that $-p_{3} \geqslant-q_{3}$ (since we now have $-p_{3} \geqslant-p_{2} \geqslant-p_{1}$ and $-q_{3} \geqslant-q_{2} \geqslant-q_{1}$ ) and $-p_{3}-p_{2}-p_{1} \geqslant-q_{1}-q_{2}-q_{3}$. Together, these imply that $p \succ q$.

$(\Leftarrow)$ From Theorem 2.5, $f(r) \geqslant 0$ for all $r \geqslant 1$. It is an easy fact that $p \succ q$ if and only if $-p \succ-q$, so $\tilde{f}(s) \geqslant 0$ for all $s \geqslant 1$. But this immediately implies that $f(r) \geqslant 0$ for all $0<r \leqslant 1$. Hence, $f(r) \geqslant 0$ for all $r>0$. 
It is not difficult to extend the proof of sufficiency to see that $p \succ q$ for real $n$-sequences implies that

$$
r^{p_{1}}+r^{p_{2}}+\ldots+r^{p_{n}} \geqslant r^{q_{1}}+r^{q_{2}}+\ldots+r^{q_{n}}
$$

for all $r>0$. But, the converse is not true for $n \geqslant 4$.

Example 2.9. Let $p$ be 7,4,4,1 and let $q$ be $6,6,2,2$. Then $r^{7}+2 r^{4}+r \geqslant$ $2 r^{6}+2 r^{2}$ for all $r>0$, but $p$ does not majorize $q$.

\section{NeWTON SEQUENCES AND SUFFICIENT CONDITIONS FOR MONOMIAL SUM INEQUALITIES}

Note that if a monomial sum inequality (1.7) holds for all p-Newton sequences, then it is also an inequality on nonnegative Newton sequences lying in the closure of p-Newton sequences. We note that a p-Newton sequence is necessarily unimodal: it is either (weakly) increasing, (weakly) decreasing or (weakly) increasing and then decreasing. The exponents can become (arbitrarily) negative, but once they become negative, they stay negative and become more negative. When increasing, the sequence increases at most geometrically and, when decreasing, it decreases at least geometrically.

Lemma 3.1. If $c_{j_{11}} c_{j_{12}} \ldots c_{j_{1 l_{1}}}+\ldots+c_{j_{m 1}} c_{j_{m 2}} \ldots c_{j_{m l}} \geqslant c_{i_{11}} c_{i_{12}} \ldots c_{i_{k_{1}}}+\ldots+$ $c_{i_{m 1}} c_{i_{m 2}} \ldots c_{i_{m k}}$, with indices satisfying (1.8), holds for all p-Newton sequences, then:

1. the largest (smallest) weight monomial is on the larger side of the inequality;

2. if we order the summands of the inequality by largest indices on each side, that is,

$$
i_{1 k_{1}} \leqslant i_{2 k_{2}} \leqslant \ldots \leqslant i_{m k_{m}} \text { and } \quad j_{1 l_{1}} \leqslant j_{2 l_{2}} \leqslant \ldots \leqslant j_{m l_{m}}
$$

the highest index of the $t$-th terms is on the lower side, for $t=1, \ldots, m$, i.e. $j_{t l_{t}} \leqslant i_{t k_{t}}$

3. if we order the summands of the inequality by smallest indices on each side, that is,

$$
i_{11} \leqslant i_{21} \leqslant \ldots \leqslant i_{m 1} \text { and } j_{11} \leqslant j_{21} \leqslant \ldots \leqslant j_{m 1},
$$

the lowest index of the $t$-th terms is on the lower side, for $t=1, \ldots, m$, i.e. $i_{t 1} \leqslant j_{t 1}$

4. increasing (or decreasing) all indices by 1 in an inequality results in an inequality. 
Proof. 1. It is clear evaluating the inequality at the p-Newton sequence $1, r$, $r^{2}, r^{3}, \ldots$ making $r$ big enough.

2. Note that sequences of the form $1, \ldots, 1,0, \ldots, 0$ are in the closure of $\mathrm{p}$-Newton sequences, so they verify the inequality: the sequence of p-Newton sequences

$$
\left\{1, \ldots, 1, \frac{1}{M}, \frac{1}{M^{2}}, \frac{1}{M^{3}}, \ldots,\right\}_{M=1}^{\infty}
$$

converges to $1, \ldots, 1,0, \ldots, 0$. These sequences, the ones formed with 1 's and 0 's, with the switch in the appropriate place show the statement.

3. Index complementation (see remark below) and Statement 2. from this lemma prove it.

4. This is true because a section of a p-Newton sequence is a p-Newton sequence.

Remark 3.2. By index complementation we mean replacing $c_{i}$ by $c_{n-i}$ for $i=$ $0,1, \ldots, n$. Index complementation of a p-Newton sequence results in a p-Newton sequence, though the normalization $c_{0}=1$ is lost (see comments before Lemma 11 in $[1])$.

Next, if the standard necessary conditions are met we note that if all monomials on the lower side of a proposed inequality are equal, then it is an inequality.

Theorem 3.3. If $i_{1} \leqslant \min \left\{j_{11}, \ldots, j_{m 1}\right\}, \max \left\{j_{12}, \ldots, j_{m 2}\right\} \leqslant i_{2}$, and $\sum_{k=1}^{m}\left(j_{k 1}+\right.$ $\left.j_{k 2}\right)=m\left(i_{1}+i_{2}\right)$, then the inequality

$$
c_{j_{11}} c_{j_{12}}+\ldots+c_{j_{m 1}} c_{j_{m 2}} \geqslant m c_{i_{1}} c_{i_{2}}
$$

holds for all p-Newton sequences.

Pr o of. By majorization of indices [3] we have

$$
c_{j_{11}} c_{j_{12}} c_{j_{21}} c_{j_{22}} \ldots c_{j_{m 1}} c_{j_{m 2}} \geqslant c_{i_{1}}^{m} c_{i_{2}}^{m} .
$$

Now the inequality follows from the fact that the arithmetic mean is at least the geometric mean.

The above fact can make some monomial sum p-Newton inequalities obvious when no other method does.

Using the NES, we may re-write a proposed monomial sum inequality (1.7) for Newton sequences as an exponential polynomial inequality

$$
r^{\sum_{t=1}^{l_{1}} x_{j_{1 t}}}+\ldots+r^{\sum_{t=1}^{l_{m}} x_{j_{1 t}}} \geqslant r^{\sum_{t=1}^{k_{1}} x_{i_{1}}}+\ldots+r^{\sum_{t=1}^{k_{m}} x_{i} t}
$$

in which we view $r>1$ as a variable. 
For the monomial sum inequality (1.7) to be valid for all p-Newton sequences, (3.1) must be a valid exponential polynomial inequality for every NES.

Using the fact (Theorem 2.1) that weak majorization for the exponents is necessary and sufficient for $\geqslant_{r}$ for the 2-term exponential polynomials, the main result of [1] may be re-cast and generalized in terms of majorization of Newton exponent sequences. Though this is not as combinatorially attractive as the statement of the result given in [1], it does point the way to a general sufficient condition for monomial sum inequalities, via Newton exponent sequences (1.2).

Lemma 3.4. If $c_{0}, \ldots, c_{n}$ is a $p$-Newton sequence with NES $x_{0}, \ldots, x_{n}$, then

$$
c_{j_{11}} c_{j_{12}} \ldots c_{j_{1 l_{1}}}+c_{j_{21}} c_{j_{22}} \ldots c_{j_{2 l_{2}}} \geqslant c_{i_{11}} c_{i_{12}} \ldots c_{i_{1 k_{1}}}+c_{i_{21}} c_{i_{22}} \ldots c_{i_{2 k_{2}}}
$$

if and only if

$$
\begin{aligned}
\left\{x_{j_{11}}+x_{j_{12}}+\right. & \left.\ldots+x_{j_{1 l_{1}}}, x_{j_{21}}+x_{j_{22}}+\ldots+x_{j_{2 l_{2}}}\right\} \\
& \succ\left\{x_{i_{11}}+x_{i_{12}}+\ldots+x_{i_{1 k_{1}}}, x_{i_{21}}+x_{i_{22}}+\ldots+x_{i_{2 k_{2}}}\right\} .
\end{aligned}
$$

Corollary 3.5. The monomial sum inequality (3.2) holds for all p-Newton sequences if and only if

$$
\begin{aligned}
\left\{x_{j_{11}}+x_{j_{12}}+\right. & \left.\ldots+x_{j_{1 l_{1}}}, x_{j_{21}}+x_{j_{22}}+\ldots+x_{j_{2 l_{2}}}\right\} \\
& \succ\left\{x_{i_{11}}+x_{i_{12}}+\ldots+x_{i_{1 k_{1}}}, x_{i_{21}}+x_{i_{22}}+\ldots+x_{i_{2 k_{2}}}\right\}
\end{aligned}
$$

for all NES's.

The above is an alternative to Theorem 21 of [1] in case $k_{1}=k_{2}=l_{1}=l_{2}=2$.

Since weak majorization of exponents is not necessary for exponential polynomial inequalities of more than 2 terms, we do not know if the analog to Corollary 3.5 holds for more complicated monomial sum inequalities. However, sufficiency does hold and provides a useful tool, as we will see later.

Lemma 3.6. The monomial sum inequality (1.7) holds for a p-Newton sequence $c_{0}, c_{1}, \ldots, c_{n}$, with NES $x_{0}, x_{1}, \ldots, x_{n}$ if

$$
\left\{\sum_{t=1}^{l_{1}} x_{j_{1 t}}, \ldots, \sum_{t=1}^{l_{m}} x_{j_{m t}}\right\} \succ_{w}\left\{\sum_{t=1}^{k_{1}} x_{i_{1 t}}, \ldots, \sum_{t=1}^{k_{m}} x_{i_{m t}}\right\}
$$


The above permits us to give our general result.

Theorem 3.7. The monomial sum inequality (1.7) holds for all p-Newton sequences if

$$
\left\{\sum_{t=1}^{l_{1}} x_{j_{1 t}}, \ldots, \sum_{t=1}^{l_{m}} x_{j_{m t}}\right\} \succ_{w}\left\{\sum_{t=1}^{k_{1}} x_{i_{1 t}}, \ldots, \sum_{t=1}^{k_{m}} x_{i_{m t}}\right\}
$$

for all NES's.

We do not yet know if there are valid monomial sum inequalities for which there is no weak majorization condition, as in the theorem, for some NES.

We note that, since

$$
r_{2}^{x \log r_{1} / \log r_{2}}=r_{1}^{x} \quad \text { for } r_{1}, r_{2}>1,
$$

the particular $r$, chosen to get a NES, is immaterial for whether or not the majorization condition holds.

Example 3.8. The inequality $c_{0} c_{1}+c_{0} c_{1}+c_{2} c_{2} \geqslant c_{0} c_{2}+c_{0} c_{2}+c_{0} c_{2}$ does not follow from the 22 case, but it follows from Theorem 3.3 and can also be demonstrated by majorization. Let us see $\left\{x_{0}+x_{1}, x_{0}+x_{1}, 2 x_{2}\right\} \succ_{w}\left\{x_{0}+x_{2}, x_{0}+x_{2}, x_{0}+x_{2}\right\}$. The total sum condition is verified: $x_{0}+x_{1}+x_{0}+x_{1}+2 x_{2} \geqslant 3 x_{0}+3 x_{2} \Leftrightarrow 2 x_{1} \geqslant x_{0}+x_{2}$, see (1.3). For the other two conditions, let us distinguish two cases:

$\triangleright x_{0}+x_{1} \geqslant 2 x_{2}$. We have $x_{1} \geqslant x_{2}$ because

$$
\left.\begin{array}{rl}
x_{0}+x_{1} & \geqslant 2 x_{2} \Rightarrow x_{0} \geqslant 2 x_{2}-x_{1} \\
2 x_{1} & \geqslant x_{0}+x_{2}
\end{array}\right\} \Rightarrow 2 x_{1} \geqslant 3 x_{2}-x_{1} \Leftrightarrow x_{1} \geqslant x_{2} .
$$

The two term condition holds: $2 x_{0}+2 x_{1} \geqslant 2 x_{0}+2 x_{2} \Leftrightarrow x_{1} \geqslant x_{2}$.

The one term condition holds: $x_{0}+x_{1} \geqslant x_{0}+x_{2} \Leftrightarrow x_{1} \geqslant x_{2}$.

$\triangleright 2 x_{2}>x_{0}+x_{1}$. We have $x_{1}>x_{0}$ and $x_{2}>x_{0}$ because

$$
\left.\begin{array}{c}
2 x_{2}>x_{0}+x_{1} \Rightarrow x_{2}>\frac{x_{0}+x_{1}}{2} \\
2 x_{1} \geqslant x_{0}+x_{2}
\end{array}\right\} \Rightarrow 2 x_{1}>\frac{3 x_{0}+x_{1}}{2} \Leftrightarrow x_{1}>x_{0},
$$

The two term condition holds: $2 x_{2}+x_{0}+x_{1} \geqslant 2 x_{0}+2 x_{2} \Leftrightarrow x_{1} \geqslant x_{0}$.

The one term condition holds: $2 x_{2} \geqslant x_{0}+x_{2} \Leftrightarrow x_{2} \geqslant x_{0}$. 


\section{The 222 Versus 222 inequalities for $n \leqslant 3$}

When the length of the Newton sequences, the number of factors in each monomial and the number of monomials is limited, there is a finite number of monomial sums and a finite number of possible inequalities. As we did in the case 22 , we have enumerated all the 222 possibilities for $n \leqslant 3$ and checked each of them. (This was a crucial step on the way to a theorem in the 22 case.) Many were easily ruled out (870 with total weight less or equal to 9 ), by a simple choice of Newton sequence, and many were verified as consequences of the 22 case (or in another easy way). This left, perhaps, 20-odd cases that required some work. Of course, some were ruled out by a rather thin set of Newton sequences. But, all were resolved. Here we present the table of valid inequalities. Since index complementation preserves a valid inequality, we need and do only present the ones of total weight less or equal to 9 , in order to economize on space. The ones that are easily verified from the prior case are presented in regular type-face, while the remainder are in bold face. Typical explanations are given for several of these after the table.

Given

$$
c_{j_{11}} c_{j_{12}}+c_{j_{21}} c_{j_{22}}+c_{j_{31}} c_{j_{32}} \geqslant c_{i_{11}} c_{i_{12}}+c_{i_{21}} c_{i_{22}}+c_{i_{31}} c_{i_{32}}
$$

we may assume, for convenience, without loss of generality, that

$$
\begin{aligned}
& i_{11} \leqslant i_{12}, \quad i_{21} \leqslant i_{22}, \quad i_{31} \leqslant i_{32} \\
& j_{11} \leqslant j_{12}, \quad j_{21} \leqslant j_{22}, \quad j_{31} \leqslant j_{32} .
\end{aligned}
$$

For short, in the table an inequality like (4.1) will be written as

$$
j_{11} j_{12}+j_{21} j_{22}+j_{31} j_{32} \geqslant i_{11} i_{12}+i_{21} i_{22}+i_{31} i_{32}
$$

The next table lists all the inequalities for $n \leqslant 3$ in increasing order with respect to their weights. The inequalities of weight 9 that are not self complementary, produce a new inequality of the same weight which is marked on the table as $\downarrow^{\text {ic }}$, meaning that the inequality comes from the above one by applying "index complementation".

P r o of s of the inequalities given in bold in the table:

(1) $00+11+12 \geqslant 01+02+02:\left(c_{0} c_{0}+c_{1} c_{1}\right)+c_{1} c_{2} \geqslant c_{0} c_{1}+\left(c_{0} c_{1}+c_{1} c_{2}\right) \geqslant$ $c_{0} c_{1}+c_{0} c_{2}+c_{0} c_{2}$.

(2) $00+11+22 \geqslant 01+02+03:\left(c_{0} c_{0}+c_{1} c_{1}\right)+c_{2} c_{2} \geqslant c_{0} c_{1}+\left(c_{0} c_{1}+c_{2} c_{2}\right) \geqslant$ $c_{0} c_{1}+c_{0} c_{2}+c_{0} c_{3}$.

(3) $00+11+22 \geqslant 01+02+12:\left(c_{0}-c_{1}\right)^{2}+\left(c_{0}-c_{2}\right)^{2}+\left(c_{1}-c_{2}\right)^{2} \geqslant 0$. 
weight $=\mathbf{2}$

$00+00+11 \geqslant 00+00+02$

$00+00+11 \geqslant 00+01+01$

weight $=3$

$00+00+12 \geqslant 00+00+03$

$00+01+11 \geqslant 00+01+02$

$00+01+11 \geqslant 01+01+01$ weight $=4$

$00+00+22 \geqslant 00+00+13$ $00+00+22 \geqslant 00+02+02$ $00+01+12 \geqslant 00+01+03$ $00+01+12 \geqslant 00+02+02$ $00+11+02 \geqslant 00+02+02$ $00+11+02 \geqslant 01+01+02$ $00+11+11 \geqslant 00+02+02$ $00+11+11 \geqslant 00+11+02$ $00+11+11 \geqslant 01+01+02$ $00+11+11 \geqslant 01+01+11$ $01+01+11 \geqslant 01+01+02$ weight $=\mathbf{5}$

$00+01+22 \geqslant 00+01+13$ $00+01+22 \geqslant 00+02+03$ $00+01+22 \geqslant 01+02+02$ $00+02+12 \geqslant 00+02+03$ $00+11+03 \geqslant 00+02+03$ $00+11+03 \geqslant 01+01+03$ $00+11+12 \geqslant 00+02+03$ $00+11+12 \geqslant 00+02+12$ $00+11+12 \geqslant 00+11+03$ $00+11+12 \geqslant 01+01+03$ $00+11+12 \geqslant 01+01+12$ $00+11+12 \geqslant 01+02+02$ $01+01+12 \geqslant 01+01+03$ $01+01+12 \geqslant 01+02+02$ $01+11+02 \geqslant 01+02+02$ $01+11+11 \geqslant 01+02+02$ $01+11+11 \geqslant 01+11+02$ weight $=6$

$00+00+33 \geqslant 00+03+03$ $00+01+23 \geqslant 00+03+03$ $00+02+13 \geqslant 00+03+03$ $00+02+22 \geqslant 00+02+13$ $00+02+22 \geqslant 00+03+03$ $00+02+22 \geqslant 02+02+02$ $00+11+13 \geqslant 00+02+13$ $00+11+13 \geqslant 00+03+03$ $00+11+13 \geqslant 01+01+13$ $00+11+22 \geqslant 00+02+13$ $00+11+22 \geqslant 00+02+22$ $00+11+22 \geqslant 00+03+03$ weight $=6$

$00+11+22 \geqslant 00+11+13$

$00+11+22 \geqslant 00+12+03$

$00+11+22 \geqslant 00+12+12$

$00+11+22 \geqslant 01+01+13$

$00+11+22 \geqslant 01+01+22$

$00+11+22 \geqslant 01+02+03$

$00+11+22 \geqslant 01+02+12$

$00+11+22 \geqslant 02+02+02$

$00+11+22 \geqslant 11+02+02$

$00+12+03 \geqslant 00+03+03$

$00+12+12 \geqslant 00+03+03$

$00+12+12 \geqslant 00+12+03$

$00+12+12 \geqslant 02+02+02$

$01+01+22 \geqslant 01+01+13$

$01+01+22 \geqslant 01+02+03$

$01+01+22 \geqslant 02+02+02$

$01+02+12 \geqslant 01+02+03$

$01+02+12 \geqslant 02+02+02$

$01+11+03 \geqslant 01+02+03$

$01+11+12 \geqslant 01+02+03$

$01+11+12 \geqslant 01+02+12$

$01+11+12 \geqslant 01+11+03$

$01+11+12 \geqslant 02+02+02$

$01+11+12 \geqslant 11+02+02$

$11+02+02 \geqslant 02+02+02$

$11+11+02 \geqslant 02+02+02$

$11+11+02 \geqslant 11+02+02$

$11+11+11 \geqslant 02+02+02$

$11+11+11 \geqslant 11+02+02$

$11+11+11 \geqslant 11+11+02$ weight $=7$

$00+01+33 \geqslant 01+03+03$ $00+11+23 \geqslant 00+02+23$ $00+11+23 \geqslant 00+03+13$ $00+11+23 \geqslant 01+01+23$ $00+11+23 \geqslant 01+03+03$ $00+12+13 \geqslant 00+03+13$ $00+12+22 \geqslant 00+03+13$ $00+12+22 \geqslant 00+12+13$ $00+12+22 \geqslant 00+22+03$ $00+12+22 \geqslant 02+02+03$ $00+12+22 \geqslant 02+02+12$ $00+22+03 \geqslant 00+03+13$ $00+22+03 \geqslant 02+02+03$ $01+01+23 \geqslant 01+03+03$ $01+02+13 \geqslant 01+03+03$ $01+02+22 \geqslant 01+02+13$ $01+02+22 \geqslant 01+03+03$ $01+02+22 \geqslant 02+02+03$

\section{weight $=7$}

$01+11+13 \geqslant 01+02+13$

$01+11+13 \geqslant 01+03+03$

$01+11+22 \geqslant 01+02+13$

$01+11+22 \geqslant 01+02+22$

$01+11+22 \geqslant 01+03+03$

$01+11+22 \geqslant 01+11+13$

$01+11+22 \geqslant 01+12+03$

$01+11+22 \geqslant 01+12+12$

$01+11+22 \geqslant 02+02+03$

$01+11+22 \geqslant 02+02+12$

$01+11+22 \geqslant 11+02+03$

$01+12+03 \geqslant 01+03+03$

$01+12+03 \geqslant 02+02+03$

$01+12+12 \geqslant 01+03+03$

$01+12+12 \geqslant 01+12+03$

$01+12+12 \geqslant 02+02+03$

$01+12+12 \geqslant 02+02+12$

$02+02+12 \geqslant 02+02+03$

$11+02+03 \geqslant 02+02+03$

$11+02+12 \geqslant 02+02+03$

$11+02+12 \geqslant 02+02+12$

$11+02+12 \geqslant 11+02+03$

$11+11+03 \geqslant 02+02+03$

$11+11+03 \geqslant 11+02+03$

$11+11+12 \geqslant 02+02+03$

$11+11+12 \geqslant 02+02+12$

$11+11+12 \geqslant 11+02+03$

$11+11+12 \geqslant 11+02+12$

$11+11+12 \geqslant 11+11+03$ weight $=8$

$00+02+33 \geqslant 02+03+03$

$00+11+33 \geqslant 00+02+33$

$00+11+33 \geqslant 00+13+13$

$00+11+33 \geqslant 01+01+33$

$00+11+33 \geqslant 01+03+13$

$00+11+33 \geqslant 02+03+03$

$00+11+33 \geqslant 11+03+03$

$00+12+23 \geqslant 00+03+23$

$00+12+23 \geqslant 00+13+13$

$00+12+23 \geqslant 02+03+03$

$00+22+13 \geqslant 00+13+13$

$00+22+13 \geqslant 02+02+13$

$00+22+13 \geqslant 02+03+03$

$00+22+22 \geqslant 00+13+13$

$00+22+22 \geqslant 00+22+13$

$00+22+22 \geqslant 02+02+13$

$00+22+22 \geqslant 02+02+22$

$00+22+22 \geqslant 02+03+03$

$01+02+23 \geqslant 02+03+03$

$01+11+23 \geqslant 01+02+23$ 
(4) $00+12+12 \geqslant 02+02+02$ : It follows from Theorem 3.3.

(5) $01+01+22 \geqslant 02+02+02$ : It follows from Theorem 3.3.

(6) $00+11+23 \geqslant 01+03+03:\left(c_{0} c_{0}+c_{1} c_{1}\right)+c_{2} c_{3} \geqslant c_{0} c_{1}+\left(c_{0} c_{1}+c_{2} c_{3}\right) \geqslant$ $c_{0} c_{1}+c_{0} c_{3}+c_{0} c_{3}$.

(7) $01+11+22 \geqslant 02+02+12: c_{0} c_{1}+\left(c_{1} c_{1}+c_{2} c_{2}\right) \geqslant\left(c_{0} c_{1}+c_{1} c_{2}\right)+c_{1} c_{2} \geqslant$ $c_{0} c_{2}+c_{0} c_{2}+c_{1} c_{2}$.

(8) $00+11+33 \geqslant 01+03+13:\left(c_{0}-c_{1}\right)^{2}+\left(c_{0}-c_{3}\right)^{2}+\left(c_{1}-c_{3}\right)^{2} \geqslant 0$.

(9) $00+12+23 \geqslant 02+03+03: c_{1} \geqslant \sqrt{c_{0} c_{2}} \Rightarrow c_{0}^{2}+c_{1} c_{2}+c_{2} c_{3}-c_{0} c_{2}-2 c_{0} c_{3} \geqslant$ $c_{0}^{2}+\sqrt{c_{0} c_{2}} c_{2}-c_{0} c_{2}+\left(c_{2}-2 c_{0}\right) c_{3}$. Now we distinguish two cases:

$\triangleright c_{2} \geqslant 2 c_{0}$. Then $\sqrt{c_{2}} \geqslant \sqrt{2 c_{0}} \geqslant \sqrt{c_{0}}$ and $c_{0}^{2}+\sqrt{c_{0} c_{2}} c_{2}-c_{0} c_{2}+\left(c_{2}-2 c_{0}\right) c_{3}=$ $c_{0}^{2}+\sqrt{c_{0}} c_{2}\left(\sqrt{c_{2}}-\sqrt{c_{0}}\right)+\left(c_{2}-2 c_{0}\right) c_{3} \geqslant 0$.

$\triangleright c_{2}<2 c_{0}$. Note that $c_{2}^{2} \geqslant c_{1} c_{3} \geqslant \sqrt{c_{0} c_{2}} c_{3} \Rightarrow c_{3} \leqslant c_{2} \sqrt{c_{2} / c_{0}}$. Therefore

$$
\begin{aligned}
c_{0}^{2}+\sqrt{c_{0} c_{2}} c_{2}-c_{0} c_{2}+\left(c_{2}-2 c_{0}\right) c_{3} \\
\geqslant c_{0}^{2}+\sqrt{c_{0} c_{2}} c_{2}-c_{0} c_{2}+\left(c_{2}-2 c_{0}\right) \frac{c_{2} \sqrt{c_{2}}}{\sqrt{c_{0}}} \\
=\frac{\left(\sqrt{c_{0}}+\sqrt{c_{2}}\right)\left(\sqrt{c_{0}}-\sqrt{c_{2}}\right)^{2}\left(c_{0}+\sqrt{c_{0} c_{2}}+c_{2}\right)}{\sqrt{c_{0}}} \geqslant 0 .
\end{aligned}
$$

(10) $00+22+13 \geqslant 02+03+03:\left(c_{0} c_{0}+c_{2} c_{2}\right)+c_{1} c_{3} \geqslant c_{0} c_{2}+\left(c_{0} c_{2}+c_{1} c_{3}\right) \geqslant$ $c_{0} c_{2}+c_{0} c_{3}+c_{0} c_{3}$.

(11) $00+22+22 \geqslant 02+03+03:\left(c_{0} c_{0}+c_{2} c_{2}\right)+c_{2} c_{2} \geqslant c_{0} c_{2}+\left(c_{0} c_{2}+c_{2} c_{2}\right) \geqslant$ $c_{0} c_{2}+c_{0} c_{3}+c_{0} c_{3}$.

(12) $01+12+13 \geqslant 02+03+03:\left(c_{0} c_{1}+c_{1} c_{2}\right)+c_{1} c_{3} \geqslant c_{0} c_{2}+\left(c_{0} c_{2}+c_{1} c_{3}\right) \geqslant$ $c_{0} c_{2}+c_{0} c_{3}+c_{0} c_{3}$.

(13) $00+12+33 \geqslant 02+03+13$ : It can happen (remember $p-N e w t o n$ sequences are unimodal):

$\triangleright c_{1} \geqslant c_{0}$ and $c_{2} \geqslant c_{3}: c_{0}^{2}+c_{1} c_{2}+c_{3}^{2}-c_{0} c_{2}-c_{0} c_{3}-c_{1} c_{3}=\left(c_{0}-c_{3}\right)^{2}+$ $\left(c_{1}-c_{0}\right)\left(c_{2}-c_{3}\right) \geqslant 0$.

$\triangleright c_{1} \geqslant c_{0}$ and $c_{2}<c_{3}$, or $c_{1}<c_{0}$ :

$$
\begin{aligned}
c_{0}^{2}+c_{1} c_{2}+c_{3}^{2} & -c_{0} c_{2}-c_{0} c_{3}-c_{1} c_{3}=\left(c_{1}-c_{0}\right)^{2}+\left(c_{2}-c_{1}\right)^{2}+\left(c_{3}-c_{2}\right)^{2} \\
& +2\left(c_{1}-c_{0}\right)\left(c_{2}-c_{1}\right)+2\left(c_{2}-c_{1}\right)\left(c_{3}-c_{2}\right)+\left(c_{1}-c_{0}\right)\left(c_{3}-c_{2}\right) \geqslant 0 .
\end{aligned}
$$

(14) $00+13+23 \geqslant 03+03+03$ : It follows from Theorem 3.3.

(15) $00+22+23 \geqslant 03+03+03$ : It follows from Theorem 3.3.

(16) $01+12+23 \geqslant 02+03+13$ : It can happen (remember $\mathrm{p}-$ Newton sequences are unimodal): 
weight $=8$

$01+11+23 \geqslant 01+03+13$

$01+11+23 \geqslant 02+03+03$

$01+11+23 \geqslant 11+03+03$

$01+12+13 \geqslant 01+03+13$

$01+12+13 \geqslant 02+02+13$

$01+12+13 \geqslant 02+03+03$

$01+12+22 \geqslant 01+03+13$

$01+12+22 \geqslant 01+22+03$

$01+12+22 \geqslant 01+12+13$

$01+12+22 \geqslant 02+02+13$

$01+12+22 \geqslant 02+02+22$

$01+12+22 \geqslant 02+03+03$

$01+12+22 \geqslant 02+12+03$

$01+22+03 \geqslant 01+03+13$

$01+22+03 \geqslant 02+03+03$

$02+02+13 \geqslant 02+03+03$

$02+02+22 \geqslant 02+02+13$

$02+02+22 \geqslant 02+03+03$

$02+12+03 \geqslant 02+03+03$

$02+12+12 \geqslant 02+03+03$

$02+12+12 \geqslant 02+12+03$

$11+02+13 \geqslant 02+02+13$

$11+02+13 \geqslant 02+03+03$

$11+02+13 \geqslant 11+03+03$

$11+02+22 \geqslant 02+02+13$

$11+02+22 \geqslant 02+02+22$

$11+02+22 \geqslant 02+03+03$

$11+02+22 \geqslant 02+12+03$

$11+02+22 \geqslant 11+02+13$

$11+02+22 \geqslant 02+12+12$

$11+02+22 \geqslant 11+03+03$

$11+03+03 \geqslant 02+03+03$

$11+11+13 \geqslant 02+02+13$

$11+11+13 \geqslant 02+03+03$

$11+11+13 \geqslant 11+02+13$

$11+11+13 \geqslant 11+03+03$

$11+11+22 \geqslant 02+02+13$

$11+11+22 \geqslant 02+02+22$

$11+11+22 \geqslant 02+03+03$

$11+11+22 \geqslant 02+12+03$

$11+11+22 \geqslant 11+02+13$

$11+11+22 \geqslant 11+02+22$

$11+11+22 \geqslant 02+12+12$

$11+11+22 \geqslant 11+12+03$

$11+11+22 \geqslant 11+03+03$

$11+11+22 \geqslant 11+11+13$

$11+11+22 \geqslant 11+12+12$

$11+12+03 \geqslant 02+03+03$

$11+12+03 \geqslant 02+12+03$ weight $=8$

weight $=\mathbf{9}$

$11+12+03 \geqslant 11+03+03 \quad 02+12+22 \geqslant 02+03+13$

$11+12+12 \geqslant 02+03+03 \downarrow^{\text {ic }} 11+12+13 \geqslant 02+03+13$

$11+12+12 \geqslant 02+12+03 \quad 02+12+22 \geqslant 02+22+03$

$11+12+12 \geqslant 02+12+12 \downarrow^{\text {ic }} 11+12+13 \geqslant 11+03+13$

$11+12+12 \geqslant 11+03+03 \quad 02+12+22 \geqslant 02+12+13$

$11+12+12 \geqslant 11+12+03 \downarrow^{\text {ic }} 11+12+13 \geqslant 02+12+13$

weight $=\mathbf{9}$

$02+12+22 \geqslant 03+03+03$

$00+03+33 \geqslant 03+03+03 \downarrow^{\text {ic }} 11+12+13 \geqslant 03+03+03$

$00+12+33 \geqslant 00+03+33 \quad 02+12+22 \geqslant 12+03+03$

$\mathbf{0 0}+\mathbf{1 2}+\mathbf{3 3} \geqslant \mathbf{0 2}+\mathbf{0 3}+\mathbf{1 3} \downarrow^{\text {ic }} 11+12+13 \geqslant 12+03+03$

$00+12+33 \geqslant 03+03+03 \quad 02+22+03 \geqslant 02+03+13$

$00+12+33 \geqslant 12+03+03 \downarrow^{\mathrm{ic}} 11+03+13 \geqslant 02+03+13$

$\mathbf{0 0}+\mathbf{1 3}+\mathbf{2 3} \geqslant \mathbf{0 3}+\mathbf{0 3}+\mathbf{0 3} \quad 02+22+03 \geqslant 03+03+03$

$\downarrow^{\mathrm{ic}} 01+02+33 \geqslant 03+03+03 \downarrow^{\mathrm{ic}} 11+03+13 \geqslant 03+03+03$

$00+22+23 \geqslant 00+13+23 \quad 11+12+22 \geqslant 02+03+13$

$\downarrow^{\mathrm{ic}} 01+11+33 \geqslant 01+02+33 \quad 11+12+22 \geqslant 02+22+03$

$00+22+23 \geqslant 02+02+23 \downarrow^{\text {ic }} 11+12+22 \geqslant 11+03+13$

$\downarrow^{\mathrm{ic}} 01+11+33 \geqslant 01+13+13 \quad 11+12+22 \geqslant 02+12+13$

$\mathbf{0 0}+\mathbf{2 2}+\mathbf{2 3} \geqslant \mathbf{0 3}+\mathbf{0 3}+\mathbf{0 3} \quad 11+12+22 \geqslant 02+12+22$

$\downarrow^{\mathrm{ic}} 01+11+33 \geqslant 03+03+03 \downarrow^{\mathrm{ic}} 11+12+22 \geqslant 11+12+13$

$01+03+23 \geqslant 03+03+03 \quad 11+12+22 \geqslant 03+03+03$

$01+12+23 \geqslant 01+03+23 \quad 11+12+22 \geqslant 12+03+03$

$01+12+23 \geqslant 01+13+13 \quad 11+12+22 \geqslant 12+12+03$

$\downarrow^{\mathrm{ic}} 01+12+23 \geqslant 02+02+23 \quad 11+12+22 \geqslant 12+12+12$

$\mathbf{0 1}+\mathbf{1 2}+\mathbf{2 3} \geqslant \mathbf{0 2}+\mathbf{0 3}+\mathbf{1 3} \quad 11+12+22 \geqslant 11+22+03$

$01+12+23 \geqslant 03+03+03 \quad 11+22+03 \geqslant 02+03+13$

$01+12+23 \geqslant 12+03+03$

$01+13+13 \geqslant 03+03+03$

$\downarrow^{\text {ic }} 02+02+23 \geqslant 03+03+03$

$01+22+13 \geqslant 01+13+13$

$\downarrow^{\text {ic }} 11+02+23 \geqslant 02+02+23$

$01+22+13 \geqslant 02+03+13$

$\downarrow^{\text {ic }} 11+02+23 \geqslant 02+03+13$

$01+22+13 \geqslant 03+03+03$

$\downarrow^{\text {ic }} 11+02+23 \geqslant 03+03+03$

$01+22+22 \geqslant 01+13+13$

$\downarrow^{\text {ic }} 11+11+23 \geqslant 02+02+23$

$01+22+22 \geqslant 01+22+13$

$11+22+03 \geqslant 02+22+03$

${ }^{\mathrm{c}} 11+22+03 \geqslant 11+03+13$

$11+22+03 \geqslant 03+03+03$

$11+22+03 \geqslant 12+03+03$

$11+22+03 \geqslant 12+12+03$

$12+03+03 \geqslant 03+03+03$

$12+12+03 \geqslant 03+03+03$

$12+12+03 \geqslant 12+03+03$

$12+12+12 \geqslant 03+03+03$

$12+12+12 \geqslant 12+03+03$

$12+12+12 \geqslant 12+12+03$

$\downarrow^{\text {ic }} 11+11+23 \geqslant 11+02+23$

$01+22+22 \geqslant 02+03+13$

$\downarrow^{\text {ic }} 11+11+23 \geqslant 02+03+13$

$01+22+22 \geqslant 02+22+03$

$\downarrow^{\mathrm{ic}} 11+11+23 \geqslant 11+03+13$

$01+22+22 \geqslant 03+03+03$

$\downarrow^{\text {ic }} 11+11+23 \geqslant 03+03+03$

$02+03+13 \geqslant 03+03+03$

$02+12+13 \geqslant 02+03+13$

$02+12+13 \geqslant 12+03+03$

$02+12+13 \geqslant 03+03+03$ 
$\triangleright c_{2} \geqslant c_{1}$ and $c_{3} \geqslant c_{0}$, or $c_{2} \leqslant c_{1}$ and $c_{3} \leqslant c_{0}$ : Note that $c_{1} c_{2} \geqslant c_{0} c_{3}$, then

$$
\begin{aligned}
c_{0} c_{1}+c_{1} c_{2}+c_{2} c_{3}-c_{0} c_{2}-c_{0} c_{3}-c_{1} c_{3} & \geqslant c_{0} c_{1}+c_{2} c_{3}-c_{0} c_{2}-c_{1} c_{3} \\
& =\left(c_{2}-c_{1}\right)\left(c_{3}-c_{0}\right) \geqslant 0 .
\end{aligned}
$$

$\triangleright c_{2}<c_{1}$ and $c_{3} \geqslant c_{0}$ : Because $c_{3} \leqslant c_{2}$ we have

$$
\begin{aligned}
& c_{0} c_{1}+c_{1} c_{2}+c_{2} c_{3}-c_{0} c_{2}-c_{0} c_{3}-c_{1} c_{3} \\
& =c_{1}\left(c_{2}-c_{3}\right)+c_{2}\left(c_{3}-c_{0}\right)+c_{0}\left(c_{1}-c_{3}\right) \geqslant 0 .
\end{aligned}
$$

$\triangleright c_{2} \geqslant c_{1}$ and $c_{3}<c_{0}$ : Because $c_{1} \geqslant c_{0}$ and $c_{1}^{2} \geqslant c_{0} c_{2}$ we have

$$
\begin{array}{r}
c_{0} c_{1}+c_{1} c_{2}+c_{2} c_{3}-c_{0} c_{2}-c_{0} c_{3}-c_{1} c_{3} \geqslant c_{0} c_{1}+c_{1} c_{2}+c_{2} c_{3}-c_{1}^{2}-c_{0} c_{3}-c_{1} c_{3} \\
=c_{1}\left(c_{0}-c_{3}\right)+c_{1}\left(c_{2}-c_{1}\right)+c_{3}\left(c_{2}-c_{0}\right) \geqslant 0 .
\end{array}
$$

(17) $01+13+13 \geqslant 03+03+03$ : It follows from Theorem 3.3.

(18) $01+22+13 \geqslant 03+03+03:\left(c_{0} c_{1}+c_{2} c_{2}\right)+c_{1} c_{3} \geqslant c_{0} c_{3}+\left(c_{0} c_{2}+c_{1} c_{3}\right) \geqslant$ $c_{0} c_{3}+c_{0} c_{3}+c_{0} c_{3}$ or Theorem 3.3.

(19) $01+22+22 \geqslant 03+03+03:\left(c_{0} c_{1}+c_{2} c_{2}\right)+c_{2} c_{2} \geqslant c_{0} c_{3}+\left(c_{0} c_{2}+c_{2} c_{2}\right) \geqslant$ $c_{0} c_{3}+c_{0} c_{3}+c_{0} c_{3}$ or Theorem 3.3.

We now recall the combinatorial description of the inequalities in the 22 case for comparison. Given an inequality $c_{j_{11}} c_{j_{12}}+c_{j_{21}} c_{j_{22}} \geqslant c_{i_{11}} c_{i_{12}}+c_{i_{21}} c_{i_{22}}$, we may assume

$$
\begin{aligned}
& i_{11} \leqslant i_{12}, \quad i_{21} \leqslant i_{22}, \quad i_{12} \leqslant i_{22}, \\
& j_{11} \leqslant j_{12}, \quad j_{21} \leqslant j_{22}, \quad j_{12} \leqslant j_{22} .
\end{aligned}
$$

Let $w_{i_{1}}=i_{11}+i_{12}, w_{i_{2}}=i_{21}+i_{22}, w_{j_{1}}=j_{11}+j_{12}$ and $w_{j_{2}}=j_{21}+j_{22}$ be the weights of the monomials. If $\left\{w_{j_{1}}, w_{j_{2}}\right\} \neq\left\{w_{i_{1}}, w_{i_{2}}\right\}$, then (see [1], Theorem 17)

$$
j_{12}, j_{22} \leqslant i_{12}, i_{22} \text { and } j_{11}, j_{21} \geqslant i_{11}, i_{21} \text {. }
$$

We call this condition DB, for "double between-ness".

Theorem 4.1 ([1], Theorem 21). The inequality $c_{j_{11}} c_{j_{12}}+c_{j_{21}} c_{j_{22}} \geqslant c_{i_{11}} c_{i_{12}}+$ $c_{i_{21}} c_{i_{22}}$, with indices satisfying (4.3), holds for all $p$-Newton sequences if and only if the following conditions are satisfied:

1. the weights $\left\{w_{j_{1}}, w_{j_{2}}\right\}$ majorize the weights $\left\{w_{i_{1}}, w_{i_{2}}\right\}$ and

2. either a) term-wise domination (majorization of indices) holds for a matching of left hand monomials with right-hand monomials (in case the weight pairs are equal) or b) DB holds (in case the weight pairs are different). 
It is possible to summarize the valid inequalities for $n \leqslant 3$ in the 222 case in a characterizing theorem, though it appears relatively complicated. To do this we need some terminology.

Consider the ordered pair $\left(\mathcal{C}_{j}, \mathcal{C}_{i}\right)$, where

$$
\mathcal{C}_{j}=c_{j_{11}} c_{j_{12}}+c_{j_{21}} c_{j_{22}}+c_{j_{31}} c_{j_{32}} \quad \text { and } \quad \mathcal{C}_{i}=c_{i_{11}} c_{i_{12}}+c_{i_{21}} c_{i_{22}}+c_{i_{31}} c_{i_{32}}
$$

with all indices between 0 and 3 . We say that $c_{p} c_{q}$ is between $c_{r} c_{s}$ if $\min \{p, q\} \geqslant$ $\min \{r, s\}$ and $\max \{p, q\} \leqslant \max \{r, s\}$. Then, $\mathcal{C}_{j}$ is $n_{1}-n_{2}-n_{3}$ between $\mathcal{C}_{i}$ if for some $m_{1}, c_{j_{m_{1} 1}} c_{j_{m_{1} 2}}$ is between at least $n_{1}$ terms of $\mathcal{C}_{i}$; for some $m_{2} \neq m_{1}, c_{j_{m_{2} 1}} c_{j_{m_{2} 2}}$ is between at least $n_{2}$ terms of $\mathcal{C}_{i}$; and for the remaining index $m_{3}, c_{j_{m_{3} 1}} c_{j_{m_{3}} 2}$ is between at least $n_{3}$ terms of $\mathcal{C}_{i}$.

The following two criteria will be used in the statement of our theorem. We will say that $\left(\mathcal{C}_{j}, \mathcal{C}_{i}\right)$ satisfies Criterion 1 if one of the following holds:

1. If $i_{m n}, j_{m n} \neq 3$ for all $m, n$, then

$$
\begin{cases}\left|\left\{(m, n): j_{m n}=k\right\}\right| \leqslant\left|\left\{(m, n): i_{m n}=k\right\}\right| & \text { if } k=0,2, \\ \left|\left\{(m, n): j_{m n}=k\right\}\right| \geqslant\left|\left\{(m, n): i_{m n}=k\right\}\right| & \text { if } k=1 .\end{cases}
$$

2. If $i_{m n}, j_{m n} \neq 0$ for all $m, n$, then

$$
\begin{cases}\left|\left\{(m, n): j_{m n}=k\right\}\right| \leqslant\left|\left\{(m, n): i_{m n}=k\right\}\right| & \text { if } k=1,3, \\ \left|\left\{(m, n): j_{m n}=k\right\}\right| \geqslant\left|\left\{(m, n): i_{m n}=k\right\}\right| & \text { if } k=2 .\end{cases}
$$

3. If $\{0,3\} \subset\left\{i_{m n}, j_{m n}\right\}$, then

$$
\begin{cases}\left|\left\{(m, n): j_{m n}=k\right\}\right| \leqslant\left|\left\{(m, n): i_{m n}=k\right\}\right| & \text { if } k=0,3, \\ \left|\left\{(m, n): j_{m n}=k\right\}\right| \geqslant\left|\left\{(m, n): i_{m n}=k\right\}\right| & \text { if } k=1,2 .\end{cases}
$$

We will say that $\left(\mathcal{C}_{j}, \mathcal{C}_{i}\right)$ satisfies Criterion 2 if one of the following holds:

1. If neither $\mathcal{C}_{j}$ nor $\mathcal{C}_{i}$ has repeated terms, then $\mathcal{C}_{j}$ is 2-2-3 between $\mathcal{C}_{i}$.

2. If only one of $\mathcal{C}_{j}$ and $\mathcal{C}_{i}$ has a repeated term, then $\mathcal{C}_{j}$ is 2-3-3 between $\mathcal{C}_{i}$.

3. If $\mathcal{C}_{j}$ and $\mathcal{C}_{i}$ each have a repeated term, then $\mathcal{C}_{j}$ is 3-3-3 between $\mathcal{C}_{i}$.

We may classify an ordered pair $\left(\mathcal{C}_{j}, \mathcal{C}_{i}\right)$ by the weights $w_{j_{k}}, w_{i_{k}}$ as follows. Below, brackets [ ] denote a multiset.

1. Class 1 will consist of those $\left(\mathcal{C}_{j}, \mathcal{C}_{i}\right)$ with $\left[w_{j_{k}}\right]_{k=1}^{3}=\left[w_{i_{k}}\right]_{k=1}^{3}$.

2. Class 2 will consist of those $\left(\mathcal{C}_{j}, \mathcal{C}_{i}\right)$ where $\left[w_{j_{k}}\right]_{k=1}^{3} \cap\left[w_{i_{k}}\right]_{k=1}^{3}$ contains exactly one element, say $w$. We may further subdivide this class by examining those terms in $\left(\mathcal{C}_{j}, \mathcal{C}_{i}\right)$ which have weight $w$. Even though $\mathcal{C}_{j}$ and $\mathcal{C}_{i}$ have only the 
weight $w$ in common, it may happen that other terms of $\mathcal{C}_{j}$ and $\mathcal{C}_{i}$ have this weight. For example, let $\mathcal{C}_{j}=c_{0}^{2}+c_{1}^{2}+c_{1} c_{2}$ and $\mathcal{C}_{i}=c_{0} c_{1}+c_{0} c_{2}+c_{0} c_{2}$. Here $w=2$ and it is the only weight in common, yet $\mathcal{C}_{i}$ has two terms with weight 2 . But because we only allow $0 \leqslant i_{m n}, j_{m n} \leqslant 3$, there are at most two monomials $c_{p} c_{q}$ with any particular weight. Therefore, given $\left(\mathcal{C}_{j}, \mathcal{C}_{i}\right)$ in Class 2 with common weight $w$, we have the following possibilities:

(a) $\mathcal{C}_{j}$ and $\mathcal{C}_{i}$ each has exactly one term with weight $w$.

(b) One and only one of $\mathcal{C}_{j}$ and $\mathcal{C}_{i}$ has at least two terms with weight $w$, and all of these terms are identical.

(c) One and only one of $\mathcal{C}_{j}$ and $\mathcal{C}_{i}$ has at least two terms with weight $w$, but these terms are not all the same.

In the third case, we note that $\mathcal{C}_{j}$ and $\mathcal{C}_{i}$ necessarily share a term with weight $w$, which will be called "the common-weight term" of $\mathcal{C}_{j}$ and $\mathcal{C}_{i}$. Otherwise, they each have a unique term (up to repetition) with the common weight $w$, and we may speak of "the common-weight term" of $\mathcal{C}_{j}$, i.e., the term of $\mathcal{C}_{j}$ with weight $w$, and that of $\mathcal{C}_{i}$ without ambiguity.

(a) Class 2 (a) will consist of those $\left(\mathcal{C}_{j}, \mathcal{C}_{i}\right)$ where the common-weight term of $\mathcal{C}_{j}$ and that of $\mathcal{C}_{i}$ are the same.

(b) Class 2 (b) will consist of those $\left(\mathcal{C}_{j}, \mathcal{C}_{i}\right)$ not in 2 (a) such that $\mathcal{C}_{j}$ dominates $\mathcal{C}_{i}$ by secondary term-wise domination, i.e., if $c_{j_{m 1}} c_{j_{m 2}}$ is the common-weight term of $\mathcal{C}_{j}$ and $c_{i_{n 1}} c_{i_{n 2}}$ is that of $\mathcal{C}_{i}$, we have

$$
c_{j_{m 1}} c_{j_{m 2}} \geqslant c_{i_{n 1}} c_{i_{n 2}}, \quad \sum_{k \neq m} c_{j_{k 1}} c_{j_{k 2}} \geqslant \sum_{k \neq n} c_{i_{k 1}} c_{i_{k 2}}
$$

on all p-Newton sequences.

(c) Class 2 (c) will consist of those $\left(\mathcal{C}_{j}, \mathcal{C}_{i}\right)$ not in 2 (a) and not in 2 (b) where the common-weight term of $\mathcal{C}_{j}$ dominates that of $\mathcal{C}_{i}$.

(d) Class $2(\mathrm{~d})$ will consist of those $\left(\mathcal{C}_{j}, \mathcal{C}_{i}\right)$ not in 2 (a) where the common-weight term of $\mathcal{C}_{i}$ dominates that of $\mathcal{C}_{j}$.

3. Class 3 will consist of those $\left(\mathcal{C}_{j}, \mathcal{C}_{i}\right)$ with $\left[w_{j_{k}}\right]_{k=1}^{3} \cap\left[w_{i_{k}}\right]_{k=1}^{3}=\emptyset$.

Theorem 4.2. Let

$$
\mathcal{C}_{j}=c_{j_{11}} c_{j_{12}}+c_{j_{21}} c_{j_{22}}+c_{j_{31}} c_{j_{32}} \quad \text { and } \quad \mathcal{C}_{i}=c_{i_{11}} c_{i_{12}}+c_{i_{21}} c_{i_{22}}+c_{i_{31}} c_{i_{32}}
$$

with all indices between 0 and 3 and satisfying (4.2). Then $\mathcal{C}_{j} \geqslant \mathcal{C}_{i}$ for all p-Newton sequences if and only if

(1) $\left(w_{j_{1}}, w_{j_{2}}, w_{j_{3}}\right) \succ\left(w_{i_{1}}, w_{i_{2}}, w_{i_{3}}\right)$; 
(2) if $\left(\mathcal{C}_{j}, \mathcal{C}_{i}\right)$ is in Class 1 , there is term-wise domination;

(3) if $\left(\mathcal{C}_{j}, \mathcal{C}_{i}\right)$ is in Class 2 (a) or 2 (b), $\mathcal{C}_{j}$ secondary term-wise dominates $\mathcal{C}_{i}$;

(4) if $\left(\mathcal{C}_{j}, \mathcal{C}_{i}\right)$ is in Class 2 (c) or 3 , it meets criteria 1 and 2; and

(5) $\left(\mathcal{C}_{j}, \mathcal{C}_{i}\right)$ is not in Class $2(\mathrm{~d})$.

The proof of the theorem is the inventory given in the table. The theorem may be interpreted beyond $n=3$, but, unfortunately, it does not remain valid. Item (2) is no longer necessary, as shown by Example 5.4. However, (2) does remain valid in some situations, which we explore.

\section{The EQUal Weights CASE}

In the case 22 versus 22 we must always have weight majorization, and, in the extreme case of equal weights, term-wise domination is necessary and sufficient for an inequality. According to the table, this remains the case for 222 versus 222 when $n \leqslant 3$. The weight majorization is general for 222 . What about the case of equal weights for longer Newton sequences (or more general monomial sums); is it a general principle that term-wise domination is necessary and sufficient? Under a wide variety of situations, the answer is yes, but, unfortunately, not in general, even for the 222 case. Here we give the variety of affirmative situations and then use Theorem 3.7 to verify a counterexample that may be smallest. A number of other examples may also be verified.

Theorem 5.1. If $c_{j_{11}} c_{j_{12}}+c_{j_{21}} c_{j_{22}}+c_{j_{31}} c_{j_{32}} \geqslant c_{i_{11}} c_{i_{12}}+c_{i_{21}} c_{i_{22}}+c_{i_{31}} c_{i_{32}}$, with indices satisfying (4.2), holds for all p-Newton sequences and $w_{i_{k}}=w_{j_{k}}=w$ for $k=1,2,3$, then term-wise domination holds.

Proof. Without loss of generality, we can assume that $i_{12} \leqslant i_{22} \leqslant i_{32}$ and $j_{12} \leqslant j_{22} \leqslant j_{32}$. By Lemma 3.1 we have $j_{k 2} \leqslant i_{k 2}$ for all $k$. But under the assumption of equal weights we get

$$
i_{k 1} \leqslant j_{k 1} \leqslant j_{k 2} \leqslant i_{k 2}
$$

for all $k$, or equivalently $c_{j_{k 1}} c_{j_{k 2}} \geqslant c_{i_{k 1}} c_{i_{k 2}}$ for all $k$ (see (1.1)).

Theorem 5.2. If $c_{j_{11}} c_{j_{12}}+c_{j_{21}} c_{j_{22}}+c_{j_{31}} c_{j_{32}} \geqslant c_{i_{11}} c_{i_{12}}+c_{i_{21}} c_{i_{22}}+c_{i_{31}} c_{i_{32}}$, with indices satisfying (4.2), holds for all p-Newton sequences and $\left\{w_{i_{k}}\right\}_{k=1}^{3}=\left\{w_{j_{k}}\right\}_{k=1}^{3}$ takes exactly two different values $w<w^{\prime}$, then there is term-wise domination. 
Proof. Without loss of generality, we can assume that $i_{12} \leqslant i_{22} \leqslant i_{32}$ and $j_{12} \leqslant j_{22} \leqslant j_{32}$. By Lemma 3.1 we have $j_{k 2} \leqslant i_{k 2}$ for all $k$.

Note that term-wise domination holds in $c_{j_{11}} c_{j_{12}}+c_{j_{21}} c_{j_{22}}+c_{j_{31}} c_{j_{32}} \geqslant c_{i_{11}} c_{i_{12}}+$ $c_{i_{21}} c_{i_{22}}+c_{i_{31}} c_{i_{32}}$, with the considered sequence of weights, if and only if term-wise domination holds for two terms (see (1.1)).

Suppose first that the weight $w^{\prime}$ is attained twice, i.e.,

$$
\left\{w_{i_{1}}, w_{i_{2}}, w_{i_{3}}\right\}=\left\{w_{j_{1}}, w_{j_{2}}, w_{j_{3}}\right\}=\left\{w, w^{\prime}, w^{\prime}\right\}
$$

We will prove the result matching a $j$ monomial with an $i$ monomial of equal weight and the biggest $j$-index less than or equal to biggest $i$-index. In this situation (1.1) guarantees that the $j$ monomial beats the $i$ monomial. It can happen:

$\triangleright w=w_{j_{1}}=w_{i_{1}}$. In this case $w^{\prime}=w_{j_{k}}=w_{i_{k}}$ for $k=2,3$ and the same argument as in the previous theorem proves term-wise domination.

$\triangleright w=w_{j_{1}}=w_{i_{2}}$. The matchings $w=w_{j_{1}}=w_{i_{2}}$ and $w^{\prime}=w_{j_{3}}=w_{i_{3}}$ give term-wise domination:

$$
\left.\begin{array}{c}
w=w_{j_{1}}=w_{i_{2}} \\
j_{12} \leqslant i_{22}
\end{array}\right\} \stackrel{(1.1)}{\Longrightarrow} c_{j_{11}} c_{j_{12}} \geqslant c_{i_{21}} c_{i_{22}}
$$

and

$$
\left.\begin{array}{c}
w^{\prime}=w_{j_{3}}=w_{i_{3}} \\
j_{32} \leqslant i_{32}
\end{array}\right\} \stackrel{(1.1)}{\Longrightarrow} c_{j_{31}} c_{j_{32}} \geqslant c_{i_{31}} c_{i_{32}} .
$$

$\triangleright w=w_{j_{1}}=w_{i_{3}}$. The matchings $w=w_{j_{1}}=w_{i_{3}}$ and $w^{\prime}=w_{j_{2}}=w_{i_{2}}$ give the result.

$\triangleright w=w_{j_{2}}=w_{i_{1}}$. The matchings $w^{\prime}=w_{j_{1}}=w_{i_{2}}$ and $w^{\prime}=w_{j_{3}}=w_{i_{3}}$ give the result.

$\triangleright w=w_{j_{2}}=w_{i_{2}}$. The matchings $w=w_{j_{2}}=w_{i_{2}}$ and $w^{\prime}=w_{j_{3}}=w_{i_{3}}$ give the result.

$\triangleright w=w_{j_{2}}=w_{i_{3}}$. The matchings $w=w_{j_{2}}=w_{i_{3}}$ and $w^{\prime}=w_{j_{1}}=w_{i_{1}}$ give the result.

$\triangleright w=w_{j_{3}}=w_{i_{1}}$. The matchings $w^{\prime}=w_{j_{1}}=w_{i_{2}}$ and $w^{\prime}=w_{j_{2}}=w_{i_{3}}$ give the result.

$\triangleright w=w_{j_{3}}=w_{i_{2}}$. The matchings $w^{\prime}=w_{j_{1}}=w_{i_{1}}$ and $w^{\prime}=w_{j_{2}}=w_{i_{3}}$ give the result.

$\triangleright w=w_{j_{3}}=w_{i_{3}}$. The matchings $w=w_{j_{3}}=w_{i_{3}}$ and $w^{\prime}=w_{j_{1}}=w_{i_{1}}$ give the result.

If the weight $w$ is the one attained twice, the result follows by index complementation.

Theorem 5.3. If $c_{j_{11}} c_{j_{12}}+c_{j_{21}} c_{j_{22}}+c_{j_{31}} c_{j_{32}} \geqslant c_{i_{11}} c_{i_{12}}+c_{i_{21}} c_{i_{22}}+c_{i_{31}} c_{i_{32}}$, with indices satisfying (4.2), holds for all p-Newton sequences and

$$
w_{j_{1}}<w_{j_{2}}<w_{j_{3}} \text { and } w_{i_{k}}=w_{j_{k}} \quad \text { for all } k
$$


and at least one of

$$
\begin{aligned}
& i_{11} \leqslant j_{21}<i_{21} \leqslant j_{11} \leqslant j_{12} \leqslant i_{22}<j_{22} \leqslant i_{12}, \\
& i_{31} \leqslant j_{21}<i_{21} \leqslant j_{31} \leqslant j_{32} \leqslant i_{22}<j_{22} \leqslant i_{32}
\end{aligned}
$$

is not true, then there is term-wise domination.

Proof. We will show that if there is no term-wise domination, then there is a NES such that the exponential version of (4.1) is not true for some $r>1$. It is clear that term-wise domination holds if and only if $i_{k 1} \leqslant j_{k 1} \leqslant j_{k 2} \leqslant i_{k 2}$ for all $k$. If $j_{11}<i_{11} \leqslant i_{12}<j_{12}$, then consider the sequence

$$
x_{i}= \begin{cases}-i & \text { if } i \leqslant i_{12}, \\ -i_{12}-C\left(i-i_{12}\right) & \text { if } i>i_{12},\end{cases}
$$

where $C \geqslant 1$ is a constant to be determined. Then $\beta=\max _{1 \leqslant k \leqslant 3}\left\{x_{j_{k 1}}+x_{j_{k 2}}\right\} \leqslant$ $\max \left\{-j_{11}-i_{12}-C,-w_{j_{2}},-w_{j_{3}}\right\}, \alpha=\max _{1 \leqslant k \leqslant 3}\left\{x_{i_{k 1}}+x_{i_{k 2}}\right\}=\max \left\{-w_{i_{1}}, x_{i_{21}}+\right.$ $\left.x_{i_{22}}, x_{i_{31}}+x_{i_{32}}\right\} \geqslant-w_{i_{1}}$. For sufficiently large $C, \beta \leqslant-w_{j_{2}}<-w_{i_{1}} \leqslant \alpha$. Index complementation handles $j_{31}<i_{31} \leqslant i_{32}<j_{32}$. So, suppose we have

$$
\begin{aligned}
& i_{11} \leqslant j_{11} \leqslant j_{12} \leqslant i_{12}, \\
& j_{21}<i_{21} \leqslant i_{22}<j_{22}, \\
& i_{31} \leqslant j_{31} \leqslant j_{32} \leqslant i_{32} .
\end{aligned}
$$

If $i_{12}<j_{22}$, use

$$
x_{i}= \begin{cases}-i & \text { if } i \leqslant M, \\ -M-C(i-M) & \text { if } i>M,\end{cases}
$$

where $C \geqslant 1$ will be determined and $M=j_{22}-1$. Then $\beta=\max _{1 \leqslant k \leqslant 3}\left\{x_{j_{k 1}}+\right.$ $\left.x_{j_{k 2}}\right\} \leqslant \max \left\{-w_{j_{1}},-j_{21}-M-C,-w_{j_{3}}\right\}=-w_{j_{1}} \leqslant \max \left\{-w_{i_{1}},-w_{i_{2}}, x_{i_{31}}+x_{i_{32}}\right\}=$ $\max _{1 \leqslant k \leqslant 3}\left\{x_{i_{k 1}}+x_{i_{k 2}}\right\}=\alpha$, and when $C$ is large, $\max \left\{-j_{21}-M-C,-w_{j_{3}}\right\}=-w_{j_{3}}<$ $-w_{i_{2}} \leqslant \max \left\{-w_{i_{2}}, x_{i_{31}}+x_{i_{32}}\right\}$.

If $i_{32}<j_{22}$ use the sequence

$$
x_{i}= \begin{cases}i & \text { if } i \leqslant M, \\ M-C(i-M) M & \text { if } i>M,\end{cases}
$$

where $C \geqslant 1$ and $M=j_{22}-1$. Then $\beta=\max _{1 \leqslant k \leqslant 3}\left\{x_{j_{k 1}}+x_{j_{k 2}}\right\} \leqslant \max \left\{w_{j_{1}}, j_{21}+\right.$ $\left.(1-C) M, w_{j_{3}}\right\}=w_{j_{3}} \leqslant \max \left\{x_{i_{11}}+x_{i_{12}}, w_{i_{2}}, w_{i_{3}}\right\}=\max _{1 \leqslant k \leqslant 3}\left\{x_{i_{k 1}}+x_{i_{k 2}}\right\}=\alpha$. Because $M>0$ for large $C, \max \left\{w_{j_{1}}, j_{21}+(1-C) M\right\}=w_{j_{1}}<w_{i_{2}} \leqslant \max \left\{x_{i_{11}}+\right.$ $\left.x_{i_{12}}, w_{i_{2}}\right\}$. 
If $j_{12}>i_{22}$ consider the sequence

$$
x_{i}= \begin{cases}-i & \text { if } i \leqslant i_{22}, \\ -i_{22}-C\left(i-i_{22}\right) & \text { if } i>i_{22},\end{cases}
$$

where $C \geqslant 1$. Then $\beta=\max _{1 \leqslant k \leqslant 3}\left\{x_{j_{k 1}}+x_{j_{k 2}}\right\} \leqslant \max \left\{-j_{11}-i_{22}-C,-j_{21}-\right.$ $\left.i_{22}-C,-w_{j_{3}}\right\}, \alpha=\max _{1 \leqslant k \leqslant 3}\left\{x_{i_{k 1}}+x_{i_{k 2}}\right\}=\max \left\{x_{i_{11}}+x_{i_{12}},-w_{i_{2}}, x_{i_{31}}+x_{i_{32}}\right\}$. For large enough $C$, we have $\beta \leqslant-w_{j_{3}}<-w_{i_{2}} \leqslant \alpha$.

Finally, if $j_{32}>i_{22}$ consider

$$
x_{i}= \begin{cases}i & \text { if } i \leqslant i_{22}, \\ i_{22}-C\left(i-i_{22}\right) i_{22} & \text { if } i>i_{22}\end{cases}
$$

where $C \geqslant 1$ is a constant that can be arbitrarily large. Then $\beta=\max _{1 \leqslant k \leqslant 3}\left\{x_{j_{k 1}}+\right.$ $\left.x_{j_{k 2}}\right\} \leqslant \max \left\{w_{j_{1}}, j_{21}+i_{22}-C, j_{31}+i_{22}-C\right\}, \alpha=\max _{1 \leqslant k \leqslant 3}\left\{x_{i_{k 1}}+x_{i_{k 2}}\right\}=\max \left\{x_{i_{11}}+x_{i_{12}}\right.$, $\left.w_{i_{2}}, x_{i_{31}}+x_{i_{32}}\right\}$. For large $C, \beta \leqslant w_{j_{1}}<w_{i_{2}} \leqslant \alpha$. Index complementation handles the cases $i_{31}>j_{21}, i_{11}>j_{21}, i_{21}>j_{31}, i_{21}>j_{11}$.

We speculate that if $w_{j_{1}}<w_{j_{2}}<w_{j_{3}}, w_{i_{k}}=w_{j_{k}}$ for all $k, i_{11} \leqslant j_{21}<i_{21} \leqslant$ $j_{11} \leqslant j_{12} \leqslant i_{22}<j_{22} \leqslant i_{12}$, and $i_{31} \leqslant j_{21}<i_{21} \leqslant j_{31} \leqslant j_{32} \leqslant i_{22}<j_{22} \leqslant i_{32}$, then (4.1) is true for all p-Newton sequences if and only if $\left(w_{i_{1}}, w_{i_{2}}, w_{i_{3}}\right)$ is an arithmetic progression.

Another speculation is that $w_{j_{1}}<w_{j_{2}}<w_{j_{3}}, w_{i_{k}}=w_{j_{k}}$ for all $k, i_{11} \leqslant j_{21}<$ $i_{21} \leqslant j_{11} \leqslant j_{12} \leqslant i_{22}<j_{22} \leqslant i_{12}$, and $i_{31} \leqslant j_{21}<i_{21} \leqslant j_{31} \leqslant j_{32} \leqslant i_{22}<j_{22} \leqslant i_{32}$ is sufficient for (4.1) to hold for all p-Newton sequences.

However, the problem examined in this section is quite different from that in [1], although the two are related. Even when $\left\{w_{i_{k}}\right\}_{k=1}^{3}=\left\{w_{j_{k}}\right\}_{k=1}^{3}$, (4.1) may hold for all p-Newton sequences without term-wise domination as the following example shows.

Example 5.4. We show that

$$
c_{2}^{2}+c_{1} c_{4}+c_{3}^{2} \geqslant c_{0} c_{4}+c_{2} c_{3}+c_{1} c_{5}
$$

for all p-Newton sequences $c: c_{0}, c_{1}, \ldots, c_{5}$. Note that we have equal weights: 4,5 , 6 on the two sides, but that we do not have term-wise domination, as $c_{1} c_{4}$ does not beat any term on the right. However, $c_{2}^{2} \geqslant c_{0} c_{4}$ and $c_{3}^{2} \geqslant c_{1} c_{5}$ for all p-Newton sequences.

We apply Theorem 3.7 to the NES, by showing that $\left\{2 x_{2}, x_{1}+x_{4}, 2 x_{3}\right\} \succ_{w}$ $\left\{x_{0}+x_{4}, x_{2}+x_{3}, x_{1}+x_{5}\right\}$. 
First, we make some observations about the sequence $x$.

(i) Note that $x_{k-1}+x_{k+1} \leqslant 2 x_{k}$ if and only if $x_{k+1}-x_{k} \leqslant x_{k}-x_{k-1}$. Hence, we get the chain of inequalities: $x_{1}-x_{0} \geqslant x_{2}-x_{1} \geqslant x_{3}-x_{2} \geqslant \ldots$, which elucidates the structure of $x$. We see that once $x$ decreases, it continues decreasing, i.e. if $x_{k} \geqslant x_{k+1}$, then $x_{k} \geqslant x_{k+1} \geqslant x_{k+2} \geqslant \ldots$

(ii) $x_{i}+x_{j} \geqslant x_{i-k}+x_{j+k}$ whenever $i \leqslant j$ and $k \geqslant 0$. This is equivalent to $x_{i}-x_{i-k} \geqslant x_{j+k}-x_{j}$ for $i \leqslant j$ and $k \geqslant 0$, which follows easily from the previous observation.

We have six cases to consider. First, note that

$$
\begin{aligned}
2 x_{2} & +\left(x_{1}+x_{4}\right)+2 x_{3} \geqslant\left(x_{0}+x_{4}\right)+\left(x_{2}+x_{3}\right)+\left(x_{1}+x_{5}\right) \\
& \Leftrightarrow x_{2}+x_{3} \geqslant x_{0}+x_{5},
\end{aligned}
$$

and the latter inequality follows from (ii) above.

1. Suppose $x_{0}+x_{4} \geqslant x_{2}+x_{3} \geqslant x_{1}+x_{5}$. Then we have

$$
\begin{aligned}
x_{1}+x_{3} \geqslant x_{0}+x_{4} \geqslant x_{2}+x_{3} & \Rightarrow x_{1} \geqslant x_{2} \\
& \Rightarrow x_{1} \geqslant x_{2} \geqslant x_{3} \geqslant x_{4} \geqslant x_{5} .
\end{aligned}
$$

Therefore,

$$
\begin{aligned}
2 x_{2} & \geqslant x_{1}+x_{3} \geqslant x_{1}+x_{4}=\left(x_{1}-x_{0}\right)+\left(x_{0}+x_{4}\right) \\
& \geqslant\left(x_{2}-x_{1}\right)+\left(x_{2}+x_{3}\right)=\left(2 x_{2}-x_{1}\right)+x_{3} \geqslant 2 x_{3} .
\end{aligned}
$$

Hence, $2 x_{2} \geqslant x_{1}+x_{4} \geqslant 2 x_{3}$. From (ii), we have $2 x_{2} \geqslant x_{0}+x_{4}$ and $x_{1}+x_{2} \geqslant$ $x_{0}+x_{3}$. Since

$$
2 x_{2}+\left(x_{1}+x_{4}\right) \geqslant\left(x_{0}+x_{4}\right)+\left(x_{2}+x_{3}\right) \Leftrightarrow x_{1}+x_{2} \geqslant x_{0}+x_{3},
$$

we find that $\left(2 x_{2}, x_{1}+x_{4}, 2 x_{3}\right) \succ_{w}\left(x_{0}+x_{4}, x_{2}+x_{3}, x_{1}+x_{5}\right)$.

2. Suppose $x_{0}+x_{4} \geqslant x_{1}+x_{5} \geqslant x_{2}+x_{3}$. Note that the inequalities derived above depend only on the fact that $x_{0}+x_{4} \geqslant x_{2}+x_{3}$, so we still have $2 x_{2} \geqslant$ $x_{1}+x_{4} \geqslant 2 x_{3}$. Now,

$$
2 x_{2}+\left(x_{1}+x_{4}\right) \geqslant\left(x_{0}+x_{4}\right)+\left(x_{1}+x_{5}\right) \Leftrightarrow 2 x_{2} \geqslant x_{0}+x_{5} .
$$

But, $2 x_{2} \geqslant x_{0}+x_{4} \geqslant x_{0}+x_{5}$ since $x_{1} \geqslant \ldots \geqslant x_{4} \geqslant x_{5}$. Hence,

$$
\left(2 x_{2}, x_{1}+x_{4}, 2 x_{3}\right) \succ_{w}\left(x_{0}+x_{4}, x_{2}+x_{3}, x_{1}+x_{5}\right) .
$$


3. Suppose that $x_{2}+x_{3} \geqslant x_{0}+x_{4} \geqslant x_{1}+x_{5}$.

(a) If $x_{2} \geqslant x_{3}$, then $2 x_{2} \geqslant 2 x_{3}$ and $x_{2} \geqslant x_{3} \geqslant x_{4} \geqslant x_{5}$. We also get

$$
2 x_{2} \geqslant x_{1}+x_{3} \geqslant x_{1}+x_{4} \text {. }
$$

Therefore, either $2 x_{2} \geqslant x_{1}+x_{4} \geqslant 2 x_{3}$ or $2 x_{2} \geqslant 2 x_{3} \geqslant x_{1}+x_{4}$. In any event, $2 x_{2} \geqslant x_{2}+x_{3}$; furthermore,

$$
\begin{aligned}
2 x_{2}+\left(x_{1}+x_{4}\right) & \geqslant\left(x_{2}+x_{3}\right)+\left(x_{0}+x_{4}\right) \Leftrightarrow x_{1}+x_{2} \geqslant x_{0}+x_{3}, \\
2 x_{2}+2 x_{3} & \geqslant\left(x_{2}+x_{3}\right)+\left(x_{0}+x_{4}\right) \Leftrightarrow x_{2}+x_{3} \geqslant x_{0}+x_{4} .
\end{aligned}
$$

Either way, $\left(2 x_{2}, x_{1}+x_{4}, 2 x_{3}\right) \succ_{w}\left(x_{0}+x_{4}, x_{2}+x_{3}, x_{1}+x_{5}\right)$.

(b) If $x_{2} \leqslant x_{3}$, then $2 x_{2} \leqslant 2 x_{3}$ and $x_{0} \leqslant x_{1} \leqslant x_{2} \leqslant x_{3}$. Therefore,

$$
2 x_{3} \geqslant x_{2}+x_{4} \geqslant x_{1}+x_{4},
$$

and either $2 x_{3} \geqslant x_{1}+x_{4} \geqslant 2 x_{2}$ or $2 x_{3} \geqslant 2 x_{2} \geqslant x_{1}+x_{4}$. In both cases, $2 x_{3} \geqslant x_{2}+x_{3} ;$ also,

$$
\begin{aligned}
2 x_{3}+\left(x_{1}+x_{4}\right) & \geqslant\left(x_{2}+x_{3}\right)+\left(x_{0}+x_{4}\right) \Leftrightarrow x_{1}+x_{3} \geqslant x_{0}+x_{2}, \\
2 x_{3}+2 x_{2} & \geqslant\left(x_{2}+x_{3}\right)+\left(x_{0}+x_{4}\right) \Leftrightarrow x_{2}+x_{3} \geqslant x_{0}+x_{4} .
\end{aligned}
$$

Hence, $\left(2 x_{2}, x_{1}+x_{4}, 2 x_{3}\right) \succ_{w}\left(x_{0}+x_{4}, x_{2}+x_{3}, x_{1}+x_{5}\right)$.

4. Suppose that $x_{2}+x_{3} \geqslant x_{1}+x_{5} \geqslant x_{0}+x_{4}$. Let $y=\left(y_{0}, y_{1}, \ldots, y_{5}\right)$ be the sequence with $y_{k}=x_{5-k}$ so that $y_{k-1}+y_{k+1} \leqslant 2 y_{k}$ for all $k$. Our hypothesis then gives $y_{3}+y_{2} \geqslant y_{4}+y_{0} \geqslant y_{5}+y_{1}$; from (3) we know that $\left(2 y_{2}, y_{1}+y_{4}, 2 y_{3}\right) \succ_{w}$ $\left(y_{0}+y_{4}, y_{2}+y_{3}, y_{1}+y_{5}\right)$, i.e. $\left(2 x_{3}, x_{4}+x_{1}, 2 x_{2}\right) \succ_{w}\left(x_{5}+x_{1}, x_{3}+x_{2}, x_{4}+x_{0}\right)$.

The remaining two cases, $x_{1}+x_{5} \geqslant x_{0}+x_{4} \geqslant x_{2}+x_{3}$ and $x_{1}+x_{5} \geqslant x_{2}+x_{3} \geqslant$ $x_{0}+x_{4}$, can be argued as in 4 .

\section{ADDENDUM}

In prior paper [1], in the case of inequalities between one sum of two two-factor monomials and another (22), a principle is given as a lemma (Lemma 19), in which increasing by 1 the higher index in each term preserves valid inequalities. (It is equivalent to decreasing the lower index in each term by 1.) The principle, stated there only for the indicated 22 versus 22 case, is valid, but the proof is not complete, and it contains an inadvertent misstatement ("more" should be "less"). For the record, we give here a complete, properly stated proof. The statement of the lemma, the notation and references, as well as the comment about spreading indices, are exactly the same. 
P r o of (of Lemma 19 in [1]). In the case of term-wise domination, the statement is trivially correct, monomial by monomial. So, we may assume that there is no termwise domination, in which case we know that there must be double between-ness in the hypothesized valid inequality by Theorem 17 (display (13)) of [1].

Now,

$$
\begin{aligned}
c_{j_{11}} c_{j_{12}+1}+c_{j_{21}} c_{j_{22}+1} & =c_{j_{11}} c_{j_{12}} \frac{c_{j_{12}+1}}{c_{j_{12}}}+c_{j_{21}} c_{j_{22}} \frac{c_{j_{22}+1}}{c_{j_{22}}} \\
& \geqslant\left(c_{j_{11}} c_{j_{12}}+c_{j_{21}} c_{j_{22}}\right) \frac{c_{j_{22}+1}}{c_{j_{22}}} \\
& \geqslant\left(c_{i_{11}} c_{i_{12}}+c_{i_{21}} c_{i_{22}}\right) \frac{c_{j_{22}+1}}{c_{j_{22}}} \\
& =c_{i_{11}} c_{i_{12}} \frac{c_{j_{22}+1}}{c_{j_{22}}}+c_{i_{21}} c_{i_{22}} \frac{c_{j_{22}+1}}{c_{j_{22}}} \\
& \geqslant c_{i_{11}} c_{i_{12}} \frac{c_{i_{12}+1}}{c_{i_{12}}}+c_{i_{21}} c_{i_{22}} \frac{c_{i_{22}+1}}{c_{i_{22}}}=c_{i_{11}} c_{i_{12}+1}+c_{i_{21}} c_{i_{22}+1} .
\end{aligned}
$$

The first inequality holds because the ratios of consecutive elements of a p-Newton sequence form a nonincreasing sequence. The second is simply the substitution of a valid inequality. And the third holds because of double between-ness and the nonincreasing ratios property of p-Newton sequences.

The other claim, about decreeasing the smaller index in each term by 1 , follows by complementation of indices.

We note that Lemma 19 also follows from the characterization, Theorem 21. However, Lemma 19 is used (significantly) in the proof of sufficiency of double betweenness. Fortunately, only the necessity of double between-ness is used in the above proof (and that was proven as Theorem 17 of [1]), so that the overall integrity of the logic is intact.

We also note that we conjecture the principle of Lemma 19 (increasing the largest (decreasing the smallest) index in each term of a valid inequality by 1 to get a valid inequality) for general inequalities [1]. We have proven some other cases, but, so far, only when something more about the structure of the inequality is known. This is a very attractive and worthy conjecture.

Finally, we take this opportunity to note a typo in line 5 of the proof of Theorem 21 of [1]. "completation", which appears, should be "complementation". 


\section{References}

[1] C. R. Johnson, C. Marijuán, M. Pisonero: Inequalities for linear combinations of monomials in p-Newton sequences. Linear Algebra Appl. 439 (2013), 2038-2056.

[2] C.R. Johnson, C.Marijuán, M. Pisonero: Matrices and spectra satisfying the Newton inequalities. Linear Algebra Appl. 430 (2009), 3030-3046.

[3] C.R. Johnson, C. Marijuán, M. Pisonero, O. Walch: Monomials inequalities for Newton coefficients and determinantal inequalities for p-Newton matrices. Trends in Mathematics, Notions of Positivity and the Geometry of Polynomials (Brändén, Petter et al., eds.). Springer, Basel, 2011, pp. 275-282.

[4] I. Newton: Arithmetica Universalis: Sive de Compositione et Resolutione Arithmetica Liber (William Whiston, ed.). London, 1707.

[5] X. Wang: A simple proof of Descartes's rule of signs. Am. Math. Mon. 111 (2004), $525-526$.

Authors' addresses: Charles R. Johnson, Department of Mathematics, College of William and Mary, P.O. Box 8795, Williamsburg, Virginia, 23187-8795, USA, e-mail: crjohn @wm.edu; Carlos Marijuán, Departamento Matemática Aplicada, Escuela de Ingeniería Informática, Paseo de Belén 15, 47011-Valladolid, Spain, email: marijuan@mat.uva.es; Miriam P is on er o (corresponding author), Departamento Matemática Aplicada, E.T.S. de Arquitectura, Avenida de Salamanca 18, 47014-Valladolid, Spain, e-mail:mpisoner@ maf.uva.es; Michael Yeh, Purdue University, 610 Purdue Mall, West Lafayette, Indiana, 47907 USA, yehm@purdue.edu. 